\title{
Dynamic Closure of Scalar Dissipation Rate for Large Eddy Simulations of Turbulent Premixed Combustion: A Direct Numerical Simulations Analysis
}

\author{
Y. Gao $^{1} \cdot$ N. Chakraborty ${ }^{1} \cdot$ N. Swaminathan ${ }^{2}$
}

Received: 9 July 2014 / Accepted: 14 July 2015 / Published online: 21 August 2015

(C) Springer Science+Business Media Dordrecht 2015

\begin{abstract}
Dynamic algebraic closure of scalar dissipation rate (SDR) of reaction progress variable in the context of Large Eddy Simulations (LES) of turbulent premixed combustion has been addressed here using a power-law based expression and a model, which was originally proposed for Reynolds Averaged Navier Stokes (RANS) simulations, but has recently been extended for LES. The performances of these models have been assessed based on a-priori analysis of a Direct Numerical Simulations (DNS) database of statistically planar turbulent premixed flames with a range of different values of heat release parameter $\tau$, turbulent Reynolds number $R e_{t}$ and global Lewis number $L e$. It has been found that the power-law model with a single constant exponent $\alpha_{D}$ does not adequately capture the volume-averaged behaviour of density-weighted SDR and this problem is particularly severe especially for $L e<<1$ flames. The deficiency of the power-law model with a single power-law exponent arises due to multi-fractal nature of SDR. The dynamic evaluation of the model parameter for the algebraic model, which was originally proposed in the context of RANS and has been extended here for LES, has been shown to capture the local behaviour of SDR better than the power-law model. It has been demonstrated that the empirical parameterisation of a model parameter for the static version of the RANS-extended SDR model can be avoided using a dynamic formulation which captures the local behaviour of SDR either comparably or better than the static formulation for a range of different values of $\tau, L e$ and $R e_{t}$, without sacrificing the prediction of the volume-averaged SDR.
\end{abstract}

Keywords Scalar dissipation rate - Large eddy simulation · Direct numerical simulation · A-priori analysis

N. Chakraborty

nilanjan.chakraborty@newcastle.ac.uk

1 School of Mechanical and Systems Engineering, Newcastle University,

Claremont Road, Newcastle-Upon-Tyne, NE1 7RU, UK

2 Cambridge University Engineering Department, Trumpington Street, Cambridge, CB2 1PZ, UK 


\section{Introduction}

The Scalar Dissipation Rate (SDR) characterises the rate of micro-mixing in turbulent reacting flows and its importance is well-recognised for turbulent non-premixed combustion modelling and interested readers are referred to Refs. [1-4] and references therein for a detailed account of the relevance of SDR modelling in both single and multi-phase non-premixed combustion. However, the closure of SDR is relatively less common for the modelling of turbulent premixed flames where SDR plays an important role in reaction rate closure in addition to characterising the rate of micro-mixing. It has been demonstrated by Bray [5] that the mean reaction rate of reaction progress variable $c$ remains directly proportional to the Favre mean value of SDR in the context of Reynolds Averaged Navier Stokes (RANS) simulations. Recent analyses [6-8] demonstrated that the formulation by Bray [5] in the context of RANS can also be extended to model the filtered reaction rate $\overline{\dot{w}}$ using the Favre filtered SDR $\tilde{N}_{c}=\overline{\rho D \nabla c . \nabla c} / \bar{\rho}$ in Large Eddy Simulations (LES) using $\overline{\dot{w}}=2 \bar{\rho} \tilde{N}_{c} /\left(2 c_{m}-1\right)$ where $\rho$ is the gas density, $D$ is the progress variable diffusivity, $\bar{Q}$ and $\tilde{Q}=\overline{\rho Q} / \bar{\rho}$ are the LES filtered and Favre-filtered value of a general quantity $Q$, respectively, and $c_{m}=\int_{0}^{1}[c \dot{w}]_{L} f_{b}(c) d c / \int_{0}^{1}[\dot{w}]_{L} f_{b}(c) d c$ is a thermo-chemical parameter representing the reaction rate-weighted progress variable with $f_{b}(c)$ being the burning rate probability density function (pdf) and the subscript ' $L$ ' refers to the values in unstrained planar laminar premixed flames. The relation $<\dot{w}>=2<\rho>\check{N}_{c} /\left(2 c_{m}-1\right)$ (where $<Q>$ and $\breve{Q}=<\rho Q>/<\rho>$ are the Reynolds averaged and Favre averaged values of a general quantity $Q$ respectively) can be derived based on the balance of the reaction rate and scalar dissipation rate contributions to the scalar variance transport in the context of RANS for $D a>>1.0$ flames where the pdf of $c$ shows high probability of finding both unburned and completely burned gases, and small probability of finding burning mixtures [5]. Chakraborty and Cant [9] demonstrated based on scaling arguments [10] that $<\dot{w}>=2<\rho N_{c}>/\left(2 c_{m}-1\right)$ remains valid even for $D a<1$ flames as long as the flamelet assumption holds. It has been demonstrated earlier [6-8] that $\overline{\dot{w}}=2 \bar{\rho} \tilde{N}_{c} /\left(2 c_{m}-1\right)$ can be used for filtered reaction rate $\overline{\dot{w}}$ closure for $\Delta>>\delta_{t h}$. Interested readers are referred to Refs. [6-8] for further discussion on this filtered reaction rate closure. It is worth noting that $\overline{\dot{w}}=2 \bar{\rho} \tilde{N}_{c} /\left(2 c_{m}-1\right)$ becomes singular for $c_{m}=0.5$ and produces unrealistic results (i.e. $\overline{\dot{w}}<0$ ) for $c_{m}<0.5$. The thermo-chemical parameter $c_{m}$ assumes a value greater than 0.5 (usually between 0.7 and 0.9 ) for the thermo-chemistry of typical hydrogen-air and hydrocarbon-air flames where the maximum value of reaction rate takes place close to the burned gas side. Bray [5] demonstrated that the magnitude of $c_{m}$ remains insensitive to the choice of the presumed continuous function approximating $f_{b}(c)$ and $c_{m}=0.5$ is obtained when $f_{b}(c)$ is a delta function with an impulse located at $c=0.5$, which can be satisfied in an unlikely scenario where only intermediates exist without any reactants or products. In addition to the reaction rate closure, the modelling of $\tilde{N}_{c}$ plays a crucial role in the modelling of micro-mixing rate in the pdf based closures and in the modelling of sub-grid scale variance [1-4].

A number of analyses [11-27] concentrated on closures of SDR in turbulent premixed flames in the context of RANS but relatively limited effort [6-8] has been directed to the modelling of SDR for LES of turbulent premixed combustion. The combustion process takes place mostly at the sub-grid level in premixed flames, as the flame thickness often remains smaller than the filter size for most practical LES. Thus, the accuracy of combustion modelling, such as an accurate SDR based closure, is crucial for the predictive capabilities 
of LES of premixed turbulent combustion. A recent analysis [7] has demonstrated that the widely used sub-grid closure of SDR given by $\tilde{N}_{c}=\overline{\rho D \nabla c \cdot \nabla c} / \bar{\rho}=\left(\tilde{D}+D_{t}\right) \nabla \tilde{c} . \nabla \tilde{c}$, where $D_{t}$ is the eddy diffusivity, often used in LES of passive scalar mixing [28] underpredicts both local and volume-averaged $\tilde{N}_{c}$ in premixed combustion. Moreover this model does not include a chemical time scale suggesting that it lacks fundamental physics of premixed combustion. In addition, intermittency associated with flame displacement may induce additional sub-grid level fluctuations, which cannot be ignored and the SDR models for the passive scalars are not expected to capture the influences from these fluctuations.

Dunstan et al. [6] addressed the modelling of $\tilde{N}_{c}=\overline{\rho D \nabla c . \nabla c} / \bar{\rho}$ in the context of LES by extending a RANS algebraic model proposed by Kolla et al. [22] (henceforth referred to as the RANS-K model), which inherently assumes a balance of the leading order terms of SDR transport equation in an order of magnitude sense for high values of Damköhler number $D a$ (i.e. $D a>>1$ ). However, the RANS-K model [22] was strictly valid for high Damköhler number (i.e. $D a>>1$ ) unity Lewis number flames while the models considered by Dunstan et al. [6] were validated based on a single DNS dataset of a planar V-flame configuration with unity global Lewis number (i.e. $L e=\lambda / \rho c_{p}=1.0$ where $\lambda$ is the thermal conductivity and $c_{p}$ is the specific heat) and the effects of global Lewis number, heat release parameter $\tau=\left(T_{a d}-T_{0}\right) / T_{0}$ (where $T_{0}$ and $T_{a d}$ are the unburned and adiabatic flame temperatures respectively) and turbulent Reynolds number $R e_{t}$ (i.e. $R e_{t}=\rho_{0} u^{\prime} l / \mu_{0}$, where $\rho_{0}$ and $\mu_{0}$ are the unburned gas density and viscosity respectively) were not addressed. Chakraborty and Swaminathan [10] extended the RANS-K model [22] for non-unity Lewis number flames and obtained good agreement with DNS data even for small values of Damköhler number (i.e. $D a<1$ ). This RANS SDR model by Chakraborty and Swaminathan [10] (henceforth referred to as the RANS-CS model) has recently been extended for LES in Ref. [7] including the influences of heat release parameter $\tau$, global Lewis number $L e$ and turbulent Reynolds number $R e_{t}$. A-priori analysis in Ref. [7] demonstrated that the RANS-extended algebraic closure of SDR for LES (see Eq. 5i for the expression which will henceforth be referred to as the LES-G model) satisfactorily captures both volume-averaged and local behaviours of $\tilde{N}_{c}$ for a range of different filter widths $\Delta$ for flames with a range of different values of $\tau, L e$ and $R e_{t}$. However, it has been found that one of the model parameters (i.e. see $\beta_{c}$ later in Eq. 5i) in the LES-G model increases with increasing $\tau$ and an empirical parameterisation was proposed by Gao et al. [7] to account for this $\tau$ dependence. Although the LES-G model [7] performs well both based on a-priori and a-posteriori analyses [7, 8] for a constant predetermined value of $\beta_{c}$, the findings by Ma et al. [8] demonstrate that the predictions of LES depend on accurate estimation of $\beta_{c}$. A change in $\tau$ has an impact on global (e.g. flame wrinkling and turbulent flame speed) and local (e.g. flame normal acceleration and vorticity distributions) features of flame turbulence interaction and it seems that these physical mechanisms influence the numerical value of $\beta_{c}$ and the modelling of subgrid velocity fluctuation $u_{\Delta}^{\prime}$ and therefore it is beneficial if this parameter can be evaluated purely based on resolved quantities. Thus, it can be argued that a dynamic evaluation of the model parameter of the LES-G model [7] can offer some advantages over the formulation where a predetermined value of $\beta_{c}$ is used according to an empirical expression (it is indeed shown later in this paper that the dynamic model satisfactorily captures $\tilde{N}_{c}$ variation with $\tilde{c}$ for small values of $R e_{t}$ and for the flames with small $L e$, whereas the static version of the model overpredicts the mean value of $\tilde{N}_{c}$ conditional on $\tilde{c}$ for a major portion of the flame brush for large filter widths in these cases). Charlette et al. [29] used a dynamic model for the evaluation of the wrinkling factor in spite of satisfactory performance of the static model [30]. The same approach has been adopted in the current analysis. In any case, 
dynamic evaluation of $\beta_{c}$ provides an alternative method of extending the RANS-CS model [10] in comparison to the methodology described by Gao et al. [7]. Furthermore, this model parameter $\beta_{c}$ represents effects induced by flame curvature which may be scale-dependent and thus it is well suited for dynamic evaluation.

Dunstan et al. [6] and Gao et al. [7] also explored the modelling of $\tilde{N}_{c}=\overline{\rho D \nabla c . \nabla c} / \bar{\rho}$ for LES by using a power-law based approach, which demonstrated that a power-law expression with a single set of values of global power-law exponent $\alpha_{D}$ and inner cut-off scale $\eta_{i D}$ does not accurately capture the local behaviour of $\tilde{N}_{c}$ even when the model parameters are tuned to capture the volume-averaged behaviours of SDR accurately. However, a dynamic evaluation of the power-law exponent $\alpha_{D}$ based on the local resolved quantities could lead to an alternative physically feasible power-law based model expression for SDR which has not yet been assessed in existing literature, as the performance of the power-law model of SDR was found to be inadequate if a global power-law exponent $\alpha_{D}$ is assumed [6, 7]. Dynamic evaluation of power-law exponents were successfully used for the generalised Flame Surface Density (FSD) (i.e. $\Sigma_{g e n}=\overline{|\nabla c|}$ ) closure in the past $[29,31]$ and given the close relation between FSD and SDR (i.e. $\Sigma_{g e n}=\overline{\left(N_{c} / D\right)^{1 / 2}}$ ) it is worthwhile to consider if the dynamic evaluation of power-law exponent $\alpha_{D}$ could lead to a satisfactory prediction of $\tilde{N}_{c}$.

In the light of above discussion the main objectives of the present analysis are:

1. To assess the performance of a recently proposed algebraic closure of $\tilde{N}_{c}$ [7] where a model parameter is dynamically evaluated and compare its predictions with the corresponding results obtained for static model parameters.

2. To assess a power-law based closure of SDR for LES by dynamically evaluating the power-law exponent.

These objectives have been addressed here by a-priori analysis based on a single-step Arrhenius type chemistry DNS database of statistically planar turbulent premixed flames with a range of different values of $\tau, L e$ and $R e_{t}$. The rest of the paper will be organised as follows. The necessary mathematical and numerical details related to this work will be presented in the next two sections. Following this, results will be presented and subsequently discussed. Finally main findings will be summarised and conclusions will be drawn.

\section{Mathematical Background}

The generalised FSD $\Sigma_{g e n}=\overline{|\nabla c|}$ is often modelled based on a power-law expression: $\Sigma_{g e n}=|\nabla \bar{c}|\left(\Delta / \eta_{i}\right)^{D_{f}-2}$ where $\eta_{i}$ is the inner cut-off scale and $D_{f}$ is the fractal dimension of the flame surface [29-34]. Due to the close relation between $\Sigma_{g e n}=\overline{\left(N_{c} / D\right)^{1 / 2}}$ and SDR, the analogy of power-law closure for FSD has been extended in previous analyses [6, 7] for possible modelling of SDR $\tilde{N}_{c}$ :

$$
\overline{\rho N_{c}}=\bar{\rho} \tilde{N}_{c}=\bar{\rho} \tilde{D} \nabla \tilde{c} \cdot \nabla \tilde{c}\left(\frac{\Delta}{\eta_{i D}}\right)^{\alpha_{D}}
$$

where $\eta_{i D}$ is the inner cut-off scale and $\alpha_{D}$ is the power-law exponent. A-priori DNS analyses [6,7] suggested that $\eta_{i D}$ scales with thermal flame thickness $\delta_{t h}=\left(T_{a d}-\right.$ $T_{0}$ ) $/ \operatorname{Max}|\nabla T|_{L}$ (where $T$ is the instantaneous dimensional temperature and the subscript $\mathrm{L}$ refers to the unstrained premixed laminar flame condition), which is consistent with the behaviour of $\eta_{i}$ obtained previously based on experimental [31, 35] and DNS [29, 32-34] 
findings. Thus, in principle, it is possible to use Eq. 1 to model $\tilde{N}_{c}$ if $\eta_{i D}$ is taken to be $\delta_{t h}$, and $\alpha_{D}$ is appropriately parameterised. Note that a specified global value of $\alpha_{D}$ in Eq. 1 leads to unphysical small values of $\bar{\rho} \tilde{N}_{c}$ (i.e. $\bar{\rho} \tilde{N}_{c} \rightarrow 0$ ) for small filter sizes (i.e. $\Delta \rightarrow 0$ ) instead of approaching the correct asymptotic $\operatorname{limit}_{\Delta \rightarrow 0} \bar{\rho} \tilde{N}_{c}=\bar{\rho} \tilde{D} \nabla \tilde{c} . \nabla \tilde{c}=$ $\rho D \nabla c$. $\nabla c$. Dunstan et al. [6] tried to overcome this difficulty by modifying Eq. 1 into the following expression:

$$
\tilde{N}_{c}=\tilde{D} \nabla \tilde{c} . \nabla \tilde{c}\left[\exp \left(-\theta_{1} \Delta / \delta_{t h}\right)+\left[1-\exp \left(-\theta_{2} \Delta / \delta_{t h}\right)\right]\left(\Delta / \eta_{i D}\right)^{\alpha_{D}}\right]
$$

where $\theta_{1}$ and $\theta_{2}$ are the model parameters. Equation 2 ensures that $\tilde{N}_{c}$ approaches to $D \nabla c . \nabla c$ when the flow is fully resolved (i.e. $\Delta \rightarrow 0$ ), where $\lim _{\Delta \rightarrow 0} \bar{\rho} \tilde{N}_{c}=\rho D \nabla c . \nabla c$. The mathematical validation of this model was provided elsewhere [6,7], thus will not be repeated here. It has been demonstrated in Refs. [6, 7] that Eq. 2 does not adequately capture the local behaviour of $\tilde{N}_{c}$ even when the optimum values of $\theta_{1}$ and $\theta_{2}$, for which the volume-averaged values of $\bar{\rho} \tilde{N}_{c}$ is accurately captured, are used. Moreover, the performance of Eq. 2 worsens with increasing $\Delta[6,7]$. The predictions of Eq. 2 will not presented in this paper because of the aforementioned deficiencies of the static version of the power law model. Interested readers are referred to Ref. [7] for the predictions of Eq. 2 and its comparison with $\tilde{N}_{c}$ extracted from explicitly filtered DNS data.

An approach to avoid the unphysical small values of $\bar{\rho} \tilde{N}_{c}$ for $\Delta \rightarrow 0$ according to Eq. 1 is a dynamic evaluation of $\alpha_{D}$ as it (i.e. $\alpha_{D} \rightarrow 0$ ) approaches to 0 for $\Delta \rightarrow 0$. Assuming $\alpha_{D}$ does not change during test filtering operation, it is possible to evaluate the power-law exponent dynamically in the following manner:

$$
\overbrace{\rho N_{c}}=\overbrace{\bar{\rho} \tilde{N}_{c}}=\overbrace{(\bar{\rho} \tilde{D} \nabla \tilde{c} . \nabla \tilde{c})} \cdot\left(\Delta / \delta_{t h}\right)^{\alpha_{D}}=\overbrace{\bar{\rho}}^{\sim} \overbrace{\tilde{\tilde{D}}} \nabla \overbrace{\tilde{c}} \cdot \nabla \overbrace{\tilde{c}} \cdot \overbrace{(\bar{\Delta}} / \delta_{t h})^{\alpha_{D}}
$$

where $\overbrace{\bar{Q}}$ and $\overbrace{\tilde{Q}}$ indicate test filtered value of a general quantity $Q$ and Favre filtering operation at the equivalent filter width (i.e. $\overbrace{\tilde{Q}}=\overbrace{\rho \overline{\rho Q}} / \overbrace{\bar{\rho}}$ ) respectively, whereas the equivalent filter width after test filtering is given by $\overbrace{\bar{\Delta}}$. The test filter is often taken to be a multiplier of $\Delta$ (i.e. $\overbrace{\Delta}=a \Delta$ where $a>1$ is a constant. Here $a$ is taken to be 2.0) for non-zero filter widths. Thus the equivalent filter width $\overbrace{\bar{\Delta}}$ for a Gaussian filter can be given as: $\overbrace{\bar{\Delta}}=\left[\Delta^{2}+(\overbrace{\Delta})^{2}\right]^{1 / 2}=\Delta \sqrt{1+a^{2}}$ [36] (i.e. $\lim _{\Delta \rightarrow 0} \overbrace{\bar{\Delta}} / \Delta=\sqrt{1+a^{2}}$ ). Therefore the ratio between $\overbrace{\bar{\Delta}}$ and $\Delta$ should be taken as a constant value. Based on Eq. 3 , it is possible to obtain an expression of $\alpha_{D}$ in the following manner:

$$
\alpha_{D}=\frac{\ln [<\overbrace{(\bar{\rho} \tilde{D} \nabla \tilde{c} . \nabla \tilde{c})}>_{D} /<\overbrace{\bar{\rho}} \overbrace{\tilde{D}} \nabla \overbrace{\tilde{c}} . \nabla \overbrace{\tilde{c}}>_{D}]}{\ln (\Delta)} \text { for } \Delta>0 \text {; otherwise } \alpha_{D}=0
$$

where $<Q>_{D}$ is an appropriate volume-averaging operation to avoid unphysical numerical artefacts induced by dynamic filtering operation [29, 31]. ${ }^{1}$ Equation 4 relies on the

\footnotetext{
${ }^{1}$ In this paper $<\ldots>_{D}$ is used to refer to ensemble averaging of the relevant quantity over a representative volume around a grid point for the purpose of avoiding unphysical numerical artefacts induced by dynamic filtering operation $[29,31]$. By contrast, $\langle\ldots\rangle_{V}$ refers to ensemble averaging of the relevant quantity over the whole DNS domain. For a homogeneous filter (e.g. Gaussian filter) the averaging process and filtering process commute and the test filter also commutes with base filter.
} 
preservation of the functional form of the power-law between $\Delta$ and $\overbrace{\bar{\Delta}}$, and thus the validity of this modelling approach is significantly dependent on it. Furthermore, $\alpha_{D}$ approaches 0 (i.e. $\ln 1 / \ln \left(\sqrt{1+a^{2}}\right)$ ) for very small filter width (i.e. $\Delta \rightarrow 0$ ), as the numerator of Eq. 4 vanishes when $\overbrace{\bar{\rho}} \overbrace{\tilde{D} \nabla} \overbrace{\tilde{c}} . \nabla \overbrace{\tilde{c}} \rightarrow \overbrace{(\bar{\rho} \tilde{D} \nabla \tilde{c} . \nabla \tilde{c})}$.

This leads to $\tilde{N}_{c} \approx \tilde{D} \nabla \tilde{c} \cdot \nabla \tilde{c}$ when the flow becomes completely resolved (i.e. $\Delta \rightarrow 0$ ). A similar dynamic closure for FSD (i.e. $\Sigma_{g e n}=|\nabla \bar{c}|\left(\Delta S_{L} / 3 \alpha_{T 0}\right)^{\alpha_{F S D}}$ ) was proposed earlier by Knikker et al. [31] where $\alpha_{F S D}$ was evaluated using an expression similar to Eq. 4 .

Dunstan et al. [6] also discussed the possibility of extending the RANS-K model [22] for the purpose of LES. However, the RANS-K model [22] was originally proposed for high Damköhler number (i.e. $D a>>1$ ) unity Lewis number flames and it was subsequently extended by Chakraborty and Swaminathan [10] for the algebraic closure of SDR (i.e. RANS-CS model) in non-unity Lewis number flames even for small values of Damköhler number (i.e. $D a<1$ ). The RANS-CS model [10] was extended by Gao et al. [7] for the purpose of LES (i.e. LES-G model) in the following manner, accounting for the different values of $\tau, L e$ and $R e_{t}:^{2}$

$$
\tilde{N}_{c}=\tilde{D} \nabla \tilde{c} . \nabla \tilde{c}+(1-f)[\underbrace{\frac{2 K_{c}^{*} S_{L}}{L e^{1.88} \delta_{t h}}}_{I}+\underbrace{\left(C_{3}^{*}-\tau \cdot D a_{\Delta} C_{4}^{*}\right) \frac{2 u_{\Delta}^{\prime}}{3 \Delta}}_{I I}] \frac{\tilde{c}(1-\tilde{c})}{\beta_{c}}
$$

where $D a_{\Delta}=\Delta S_{L} / u_{\Delta}^{\prime} \delta_{t h}$ and $K a_{\Delta}=\left(u_{\Delta}^{\prime} / S_{L}\right)^{3 / 2}\left(\Delta / \delta_{t h}\right)^{-1 / 2}$ are the local sub-grid Damköhler and Karlovitz numbers respectively based on inertial scaling (i.e. $\varepsilon_{\Delta} \sim u_{\Delta}^{\prime 3} / \Delta$ where $\varepsilon_{\Delta}$ is the sub-grid dissipation rate of turbulent kinetic energy) and $\mu \sim \rho S_{L} \delta_{t h}[33$, $34,37], C_{3}^{*}, C_{4}^{*}$ and $\beta_{c}$ are the model parameters, $S_{L}$ is the unstrained laminar burning velocity, $f=\exp \left[-0.7\left(\Delta / \delta_{t h}\right)^{1.7}\right]$ is a bridging function [7], $u_{\Delta}^{\prime}=\left[\left(\overline{\rho u_{i} u_{i}} / \bar{\rho}-\right.\right.$ $\left.\left.\tilde{u}_{i} \tilde{u}_{i}\right) / 3\right]^{1 / 2}=\left(2 k_{s g s} / 3\right)^{1 / 2}$ is the sub-grid turbulent velocity fluctuation with $u_{i}$ and $k_{s g s}$ being the $i^{t h}$ component of fluid velocity and the sub-grid turbulent kinetic energy respectively. In Eq. 5i, $K_{c}^{*}$ is a thermo-chemical parameter given by $[10,18,22]$ :

$$
K_{c}^{*}=\frac{\delta_{t h} \int_{0}^{1}\left[\rho N_{c} \nabla \cdot \vec{u} f(c)\right]_{L} d c}{S_{L} \int_{0}^{1}\left[\rho N_{c} f(c)\right]_{L} d c}
$$

The model parameters $C_{3}^{*}$ and $C_{4}^{*}$ in Eq. $5 \mathrm{i}$ are given as [7]:

$$
C_{3}^{*}=\frac{2.0 \sqrt{K a_{\Delta}}}{1.0+\sqrt{K a_{\Delta}}}, C_{4}^{*}=\frac{1.2(1.0-\tilde{c})^{\Phi}}{L e^{2.57}\left(1+K a_{\Delta}\right)^{0.4}} \text { where } \Phi=0.2+1.5|(1.0-L e)|
$$

The RANS equivalent of the model given by Eq. 5i (i.e. RSDR-CS model) was proposed for the unresolved part of the SDR (i.e. $\tilde{N}_{c}-\tilde{D} \nabla \tilde{c} \cdot \nabla \tilde{c}$ ) and the bridging function $(1-f)$ was absent in the RANS model [10]. The RANS model was derived by equating the order of magnitudes of the leading order contributions to the transport of the unresolved part of SDR

\footnotetext{
${ }^{2}$ Here Eq. $5 \mathrm{i}$ is obtained by extending a RANS model and one of its model parameters is evaluated dynamically for LES. The same approach can be adopted any other alternative SDR models for RANS.
} 
(i.e. $\left.\breve{\varepsilon}_{c}=<\rho D \nabla(c-\breve{c}) \cdot \nabla(c-\breve{c})>/<\rho>\right)$, which is discussed in detail in Appendix A. Gao et al. [7, 38] demonstrated that the unclosed terms of the $\tilde{N}_{c}$ transport equation behaves similarly to the corresponding terms in the $\breve{\varepsilon}_{c}$ transport equation for $\Delta>\delta_{t h}$. Moreover, the leading order contributors to the $\tilde{N}_{c}$ and $\breve{\varepsilon}_{c}$ transport have the same physical origins and they scale similarly. Thus, the modelling assumptions for RANS algebraic closure of SDR can also be extended to LES [7, 38]. The discussion in Appendix A suggests that the terms $I$ and $I I$ originate from the model expressions for the unresolved components of $-2<(D / \rho)[\dot{w}+\nabla \cdot(\rho D \nabla c)] \nabla c \cdot \nabla \rho>$ and $-2<\rho D\left(\partial c / \partial x_{i}\right)\left(\partial u_{i} / \partial x_{j}\right)\left(\partial c / \partial x_{j}\right)>$ respectively. The Lewis number dependence of $I$ mimics the strengthening of thermal expansion (or dilatation rate) effects with decreasing $L e$, which leads to an increase in the magnitude of $-2<(D / \rho)[\dot{w}+\nabla \cdot(\rho D \nabla c)] \nabla c \cdot \nabla \rho>$ with a decrease in Le [24]. The terms involving $C_{3}^{*}\left(C_{4}^{*}\right)$ account for alignment of $\nabla c$ with the most compressive (extensive) principal strain rates under the action of turbulent straining (flame normal acceleration) [16-18,24]. The Lewis number dependence of $C_{4}^{*}$ accounts for strengthening of flame normal acceleration due to strong heat release for small values of $L e$ (i.e. $L e ~<<1$ ) as a result of thermo-diffusive instabilities. The exponents of $L e$ in the term $I$ and $C_{4}^{*}$ are combined artefacts of the scaling $\delta_{t h} / \delta_{L} \sim L e$ (where $\delta_{L}=1 / \max |\nabla c|_{L}$ is an alternative flame thickness) and empirical fitting of DNS data. The terms $2 K_{c}^{*} S_{L} /\left(L e^{1.88} \delta_{t h}\right)$ and $-2 \tau . D a_{\Delta} u_{\Delta}^{\prime} C_{4}^{*} / 3 \Delta$ in Eq. $5 \mathrm{i}$ account for chemical time scale dependence of SDR, whereas $2 u_{\Delta}^{\prime} C_{3}^{*} / 3 \Delta$ addresses turbulent time scale dependence of SDR as in the case of passive scalar mixing. Interested readers are referred to Refs. [10,24] for more information on these derivations.

For large values of Karlovitz number, premixed turbulent flames show attributes of the broken reaction zones regime [39] combustion where the effects of heat release and flame normal acceleration are weak, and turbulent straining principally governs the behaviour of $-2<\rho D\left(\partial c / \partial x_{i}\right)\left(\partial u_{i} / \partial x_{j}\right)\left(\partial c / \partial x_{j}\right)>$. The scalar gradient $\nabla c$ aligns with the most compressive principal strain rate when turbulent straining dominates over the strain rate arising from flame normal acceleration and vice versa $[16,23,40]$. The strengthening of turbulent straining and weakening of the strain rate induced by flame normal acceleration with increasing Karlovitz number are mimicked by the empirical $K a_{\Delta}$ dependences in $C_{3}^{*}$ and $C_{4}^{*}$ respectively [14-16,19-21]. Any other functions, which account for the asymptotic behaviour of $C_{3}^{*}$ and $C_{4}^{*}$ in relation to Karlovitz number variation, can also be used in principle to parameterise these model parameters. Interested readers are referred to Refs. [10, 16-18, 22-24] for further information on the modelling of terms $I$ and $I I$, and the derivation of the RANS-K and RANS-CS models.

Here the expressions of $C_{3}^{*}, C_{4}^{*}$ and $\Phi$ are extended directly from the RANS-CS model [10] by replacing the ratio of dissipation rate of turbulent kinetic energy to turbulent kinetic energy in RANS by $u_{\Delta}^{\prime} / \Delta$ in the context of LES. The model parameter $\beta_{c}$ was modified in Refs. [6, 7] for the purpose of extending the RANS-CS model [10] for LES. The model parameters $C_{3}^{*}$ and $C_{4}^{*}$ are three-dimensional variables in the context of LES due to their dependence on $u_{\Delta}^{\prime}$, so the model parameter $\beta_{c}$ is considered for dynamic evaluation for the sake of simplicity of modelling. Also, this model parameter is influenced by scale dependent features such as flame curvature [18, 24, 25], which makes this parameter suited for dynamic evaluation. Moreover, this makes it convenient to analyse the influence of $\beta_{c}$ on SDR $\tilde{N}_{c}$ prediction independent of turbulence modelling (unlike $C_{3}^{*}$ and $C_{4}^{*}$, because the modelling of $u_{\Delta}^{\prime}$ is likely to affect these parameters). Gao et al. [7] recently demonstrated based on a-priori DNS analysis that the model parameter $\beta_{c}$ in Eq. 5i increases with increasing $\tau$, whereas $\beta_{c}$ remains insensitive to the changes in Lewis number $L e$ and turbulent Reynolds 
number $R e_{t}$. Gao et al. [7] proposed the following empirical parameterisation of $\beta_{c}$ based on a-priori DNS analysis when $u_{\Delta}^{\prime}$ is extracted from DNS data:

$$
\beta_{c}=\max \left\{\frac{2}{2 c_{m}-1},\left[\frac{1.05 \tau}{(1+\tau)}+0.51\right]^{4.6}\right\}
$$

In Eq. 5iv the minimum value of $\beta_{c}$ has been set to be $2 /\left(2 c_{m}-1\right)$ in order to satisfy the physical realisability (i.e. $\tilde{N}_{c} \geq 0$ ) according to the previous analysis by Chakraborty et al. [18]. Equation 5iv further suggests that an asymptotic value of $\beta_{c}$ can be obtained for large values of $\tau$ (i.e. $\tau \rightarrow \infty$ ). The SDR model given by Eq. $5 \mathrm{i}$ with a predetermined $\beta_{c}$ has recently been implemented in LES simulations $[8,41]$ of flow configurations for which well-documented experimental data is available for a direct comparison with simulation results and the results have been found to be either comparable or better than that obtained from established algebraic LES-FSD closures. Interested readers are referred to Ma et al. [8] for further discussion in this regard. However, Gao et al. [7] and Ma et al. [8] demonstrated that the modelling of $u_{\Delta}^{\prime}$ influences the optimum value and the parameterization of $\beta_{c}$. The empirical parameterisation of $\beta_{c}$ can be avoided using a dynamic formulation which is proposed here in the following manner. Equation 5 i can be rewritten as:

$$
\bar{\rho} \tilde{N}_{c}-\bar{\rho} \tilde{D} \nabla \tilde{c} . \nabla \tilde{c}=f_{1}\left(\frac{u_{\Delta}^{\prime}}{S_{L}}, \frac{\Delta}{\delta_{t h}}\right) \frac{\bar{\rho}}{\beta_{c}}
$$

where $f_{1}\left(\frac{u_{\Delta}^{\prime}}{S_{L}}, \frac{\Delta}{\delta_{t h}}\right)$ is given by:

$$
f_{1}\left(\frac{u_{\Delta}^{\prime}}{S_{L}}, \frac{\Delta}{\delta_{t h}}\right)=\left[1-\exp \left[-\theta\left(\frac{\Delta}{\delta_{t h}}\right)^{p}\right]\right]\left[\frac{2 K_{c}^{*} S_{L}}{L e^{1.88} \delta_{t h}}+\left(C_{3}^{*}-\tau \cdot D a_{\Delta} C_{4}^{*}\right)\left(\frac{2 u_{\Delta}^{\prime}}{3 \Delta}\right)\right] \tilde{c}(1-\tilde{c})
$$

Based on D' Germano's identity $\overbrace{\bar{\rho} \tilde{N}_{c}}=\overbrace{\overline{\rho N_{c}}}$ and the assumption of the preservation of the functional form given by Eq. 5i, one can write:

$$
\begin{aligned}
& \overbrace{\bar{\rho} \tilde{N}_{c}}-\overbrace{\bar{\rho} \tilde{D} \nabla \tilde{c} \cdot \nabla \tilde{c}}=\frac{1}{\beta_{c}} \overbrace{\bar{\rho} f_{1}\left(\frac{u_{\Delta}^{\prime}}{S_{L}}, \frac{\Delta}{\delta_{t h}}\right)}, \\
& \overbrace{\rho N_{c}}-\overbrace{\bar{\rho}} \overbrace{\tilde{D}} \nabla \overbrace{\tilde{c}} . \nabla \overbrace{\tilde{c}}=\overbrace{\frac{\bar{\rho}}{\beta_{c}}} f_{1}(\overbrace{\frac{u_{\Delta}^{\prime}}{S_{L}}} \overbrace{\bar{\Delta}})
\end{aligned}
$$

and

$$
\begin{aligned}
& f_{1}(\overbrace{\frac{u_{\Delta}^{\prime}}{S_{L}}}^{-}, \frac{\overbrace{\bar{\Delta}}}{\delta_{t h}})=\left[1-\exp \left[-\theta(\overbrace{\bar{\Delta}}^{\delta_{t h}})^{p}\right]\right] \\
& \left.[\frac{2 K_{c}^{*} S_{L}}{L e^{1.88} \delta_{t h}}+\overbrace{C_{3}^{*}}-\tau \cdot \overbrace{D a_{\Delta}} \overbrace{C_{4}^{*}}) \frac{2 \overbrace{u_{\Delta}^{\prime}}}{3 \overbrace{\bar{\Delta}}}\right] \overbrace{\tilde{c}} .(1-\overbrace{\tilde{c}})
\end{aligned}
$$


where $\overbrace{C_{3}^{*}}, \overbrace{C_{4}^{*}}$ and $\overbrace{D a_{\Delta}}$ are given by:

$$
\begin{aligned}
\overbrace{C_{3}^{*}} & =\frac{2.0(\overbrace{K a_{\Delta}})^{1 / 2}}{1+(\overbrace{K a_{\Delta}})^{1 / 2}}, \quad \overbrace{C_{4}^{*}} \\
& =\frac{1.2(1.0-\overbrace{\tilde{c}})^{\Phi}}{L e^{2.57}(1+\overbrace{K a_{\Delta}})^{0.4}} \text { and } \overbrace{D a_{\Delta}}=\overbrace{\overbrace{u_{\Delta}^{\prime}}^{\prime}}^{S_{L}} \frac{S_{L}}{\delta_{t h}}
\end{aligned}
$$

In Eq. 6v $\overbrace{K a_{\Delta}}$ and $\overbrace{u_{\Delta}^{\prime}}$ are given by:

$$
\overbrace{K a_{\Delta}}=(\overbrace{\frac{u_{\Delta}^{\prime}}{S_{L}}})^{1.5}(\overbrace{\frac{\bar{\Delta}}{\delta_{t h}}})^{-0.5} \text { and } \overbrace{u_{\Delta}^{\prime}}=\left[\frac{1}{3}(\overbrace{\frac{\overbrace{\overline{\rho u_{i} u_{i}}}}{\overbrace{\bar{\rho}}}}-\overbrace{\tilde{u}_{i}} \overbrace{\tilde{u}_{i}})\right]^{0.5}
$$

As the volume-averaged value of the density-weighted $\operatorname{SDR}\left(=\bar{\rho} \tilde{N}_{c}\right)$ should be independent of $\Delta$ (i.e. $<\overbrace{\bar{\rho} \tilde{N}_{c}}>_{V}=<\overbrace{\rho N_{c}}>_{V}$ ), which can be utilised along with Eqs. 6iii and 6iv to obtain the following dynamic evaluation of $\beta_{c}$ :

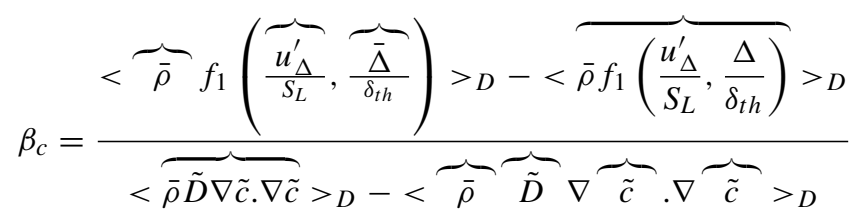

Chakraborty et al. [18] demonstrated that $\beta_{c}$ needs to satisfy $\beta_{c} \geq 2 /\left(2 c_{m}-1\right)$ in order to maintain physical realisability (i.e. $\tilde{N}_{c} \geq 0$ ) and thus it is ensured that dynamic evaluation of $\beta_{c}$ does not violate physical realisability in the following manner:

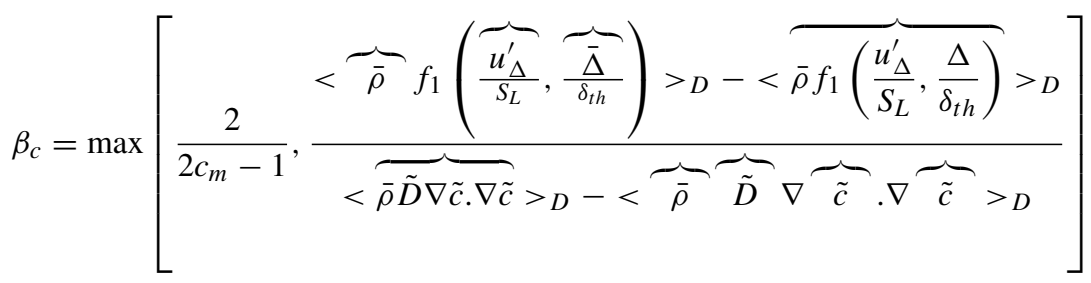

The predictions of Eqs. 1 and 5 i for dynamic evaluation of $\alpha_{D}$ and $\beta_{c}$, respectively, will be assessed with respect to explicitly filtered DNS data Section 3 of this paper. The predictions of Eq. 5 i with dynamic evaluation of $\beta_{c}$ (i.e. Eq. 7) will also be compared to the prediction of the static version of this model where $\beta_{c}$ is evaluated using Eq. 5iv. It is shown by Gao et al. [7] that modelling the sub-grid scale velocity fluctuation by $u_{\Delta}^{\prime}=$ $v_{t} /\left(C_{v} \Delta\right)$ (with $\left.C_{v}=0.094\right)$ [36] does not significantly affect the performance of the model given by Eq. $5 \mathrm{i}$ but it slightly modifies the optimum value of $\beta_{c}$ and its parameterization. Here it has also been found that modelling $u_{\Delta}^{\prime}$ by $u_{\Delta}^{\prime}=v_{t} /\left(C_{v} \Delta\right)$ does not significantly change the performance of the dynamic model in comparison to the situation when $u_{\Delta}^{\prime}$ is extracted from DNS data using $u_{\Delta}^{\prime}=\left[\left(\overline{\rho u_{i} u_{i}} / \bar{\rho}-\tilde{u}_{i} \tilde{u}_{i}\right) / 3\right]^{1 / 2}$. Thus, the results obtained using $u_{\Delta}^{\prime}=\left[\left(\overline{\rho u_{i} u_{i}} / \bar{\rho}-\tilde{u}_{i} \tilde{u}_{i}\right) / 3\right]^{1 / 2}$ extracted from DNS are only shown in the paper for the sake of conciseness. 
For passive scalar mixing $\tilde{N}_{c}=\left(\tilde{D}+D_{t}\right) \nabla \tilde{c}$. $\nabla \tilde{c}$ (where $D_{t}$ is the eddy diffusivity) is often used for SDR closure. It is shown in Ref. [7] that the conventional SDR model $\tilde{N}_{c}=\left(\tilde{D}+D_{t}\right) \nabla \tilde{c}$. $\nabla \tilde{c}$ significantly underpredicts the SDR obtained from DNS data for large filter widths (i.e. $\Delta>\delta_{t h}$ ) (see Figs. 5-7 in Ref. [7]). As the performance of $\tilde{N}_{c}=$ $\left(\tilde{D}+D_{t}\right) \nabla \tilde{c} . \nabla \tilde{c}$ remains inferior to the static versions of the power-law and extended RANS models (i.e. Eqs. 2 and 5i) and this conventional SDR model for passive scalars completely ignores chemical time scale dependence of SDR, which is essential for SDR modelling in premixed flames, this closure for passive scalars is not considered in the current analysis.

\section{Numerical Implementation}

Three dimensional DNS simulations with detailed chemistry are still extremely expensive [42] for the purpose of an extensive parametric analysis involving a range of different values of heat release parameter $\tau$, Lewis number $L e$ and turbulent Reynolds number $R e_{t}$, in particular when the DNS dataset covering a range of parameters need to be explicitly filtered over a range of different filter widths $\Delta$, as done in the current analysis. Here a simple chemistry three-dimensional DNS database has been considered where a single-step Arrhenius type irreversible chemical reaction (i.e. Reactants $\rightarrow$ Products) represents the chemical processes in the flame. It was demonstrated in Ref. [43] based on three-dimensional detailed chemistry based DNS data that the SDR transport statistics for major species are qualitatively similar to that of SDR of reaction progress variable $c$ obtained from simple chemistry DNS data. The reaction progress variable $c$ is defined based on a suitable reactant mass fraction in the following manner: $c=\left(Y_{R 0}-Y_{R}\right) /\left(Y_{R 0}-Y_{R \infty}\right)$ where $Y_{R 0}$ and $Y_{R \infty}$ denote the reactant mass fractions in unburned and fully burned gases respectively. A wellknown three-dimensional compressible DNS code SENGA [44] has been used to generate the DNS database where conservation equations of mass, momentum, energy and reaction progress variable are solved in non-dimensional form [45]. The relevant non-dimensional conservation equations and non-dimensional reaction rate of progress variable are provided in Appendix B for the sake of completeness. For the current analysis standard values are chosen for Prandtl number $\operatorname{Pr}$, Zel'dovich number $\beta=E_{a c}\left(T_{a d}-T_{0}\right) / R T_{a d}^{2}$ and ratio of specific heats $\gamma=C_{p} / C_{v}$ (i.e. $\operatorname{Pr}=0.7, \beta=6.0$ and $\gamma=1.4$ ), where $E_{a c}$ is the activation energy, $R$ is the gas constant, and $C_{p}$ and $C_{v}$ are the specific heat capacities for constant pressure and constant volume respectively. The flame Mach number $M a=S_{L} / a_{0}$ is taken to be 0.014 for all cases where $a_{0}$ is the acoustic speed in the unburned gas.

The DNS cases considered here have been used extensively in several previous publications $[9,10,24,26,46-48]$ to analyse different aspects of turbulent premixed combustion and interested readers are referred to these publications for further information regarding these cases and for the conditions under which statistics were extracted. The contours of $c$ for the cases considered here, have been presented elsewhere $[9,10,17,24,26,46$, $48]$ and thus are not repeated here. The computational domain is taken to be a rectangular parallelepiped of size $24.1 \delta_{t h} \times 24.1 \delta_{t h} \times 24.1 \delta_{t h}\left(36.1 \delta_{t h} \times 24.1 \delta_{t h} \times 24.1 \delta_{t h}\right)$ for cases A-F (G-K), which has been discretised by using a uniform Cartesian grid of $230 \times 230 \times 230(345 \times 230 \times 230)$, ensuring about 10 grid points within $\delta_{t h}$. The domain boundaries in the direction of mean flame propagation are taken as partially non-reflecting, whereas the boundaries in the transverse directions are considered to be periodic. The spatial discretisation is accounted for by a $10^{\text {th }}$ order central difference scheme for internal grid points and the order of differentiation gradually drops to a one-sided $2^{\text {nd }}$ order scheme at 
non-periodic boundaries. The time-advancement is carried out in an explicit manner using a low-storage $3^{\text {rd }}$ order Runge-Kutta scheme.

The initial values of the root-mean-square (rms) turbulent velocity fluctuation normalised by unstrained laminar burning velocity $u^{\prime} / S_{L}$ and the integral length scale to flame thickness ratio $l / \delta_{t h}$ are presented in Table 1 along with the initial values of Damköhler number $D a=$ $l S_{L} / u^{\prime} \delta_{t h}$, Karlovitz number $K a=\left(u^{\prime} / S_{L}\right)^{3 / 2}\left(l / \delta_{t h}\right)^{-1 / 2}, R e_{t}, \tau, L e, c_{m}$ and $K_{c}^{*} / \tau$. It can be seen from Table 1 that $R e_{t}$ values are comparable for cases A-F and I. The values of heat release parameter $\tau$ is 3.0 (4.5) for case A (cases B-K), whereas $L e=0.34,0.6,0.8$ and 1.2 for cases B, C, D and F respectively and in other cases $L e$ is taken to be unity. In cases G$\mathrm{K}$ the variation of $R e_{t}$ is brought about by changing either $D a$ or $K a$ independently of the other, since $R e_{t}$ scales as $R e_{t} \sim D a^{2} K a^{2}$ [39] for unity Lewis number flames.

In all cases flame-turbulence interaction takes place under decaying turbulence, which necessitates the simulation time $t_{\text {sim }} \geq \operatorname{Max}\left(t_{f}, t_{c}\right)$ [49] (where $t_{f}=l / u^{\prime}$ is the initial eddy turn over time and $t_{c}=\delta_{t h} / S_{L}$ is the chemical time scale) in order to ensure that the simulation results are independent of initialisation of chemistry and turbulent flow field. In all cases statistics were extracted after one chemical time scale $t_{c}$, which corresponds to a time equal to $2.0 t_{f}$ in case $\mathrm{J}, 3.0 t_{f}$ in cases A-F, G, I and $\mathrm{K}$, and $4.34 t_{f}$ for case $\mathrm{H}$ respectively. The present simulation time is either comparable to or greater than that used in several previous DNS studies [29, 30, 49, 50, 52-56]. By the time the statistics were extracted, the global turbulent kinetic energy and its dissipation rate in the unburned gas ahead of the flame were no longer changing rapidly with time. The level of turbulent velocity fluctuation evaluated over the whole domain had decayed by about $50 \%, 52.66 \%, 61.11 \%, 45 \%, 24 \%$ and $34 \%$ in comparison to the initial values for cases A-F, G, H, I, J and K respectively. By contrast, the integral length scale $l$ increased by factors of between 1.5 to 2.25 for case A-K, ensuring that sufficient numbers of turbulent eddies were retained in each direction to obtain useful statistics. The conditions under which the statistics were taken for these DNS cases can be found in Ref. [48] for case A, Refs. [7-10, 24, 34, 38, 40] for cases B-G, Refs. [26, 33, 46, 47] for cases H-K.

It is worth noting that the flamelet assumption remains valid for all the unity Lewis number cases (i.e. cases B, F, H-K) considered here according to the regime diagram by Peters [39]. It is an open question if the position of the non-unity Lewis number flames can be

Table 1 Initial values of the simulation parameters corresponding to the DNS database

\begin{tabular}{llllllllll}
\hline Case & $\mathrm{u}^{\prime} / \mathrm{S}_{L}$ & $\mathrm{l} / \delta_{t h}$ & $\mathrm{Re}_{t}$ & $\mathrm{Da}$ & $\tau$ & $\mathrm{Le}$ & $\mathrm{Ka}$ & $\mathrm{c}_{m}$ & $K_{c}^{*} / \tau$ \\
\hline $\mathrm{A}$ & 7.5 & 2.45 & 47.0 & 0.33 & 3.0 & 1.0 & 13.2 & 0.85 & 0.756 \\
$\mathrm{~B}$ & 7.5 & 2.45 & 47.0 & 0.33 & 4.5 & 0.34 & 13.2 & 0.92 & 0.52 \\
$\mathrm{C}$ & 7.5 & 2.45 & 47.0 & 0.33 & 4.5 & 0.6 & 13.2 & 0.87 & 0.67 \\
$\mathrm{D}$ & 7.5 & 2.45 & 47.0 & 0.33 & 4.5 & 0.8 & 13.2 & 0.867 & 0.71 \\
$\mathrm{E}$ & 7.5 & 2.45 & 47.0 & 0.33 & 4.5 & 1.0 & 13.2 & 0.825 & 0.78 \\
$\mathrm{~F}$ & 7.5 & 2.45 & 47.0 & 0.33 & 4.5 & 1.2 & 13.2 & 0.816 & 0.79 \\
$\mathrm{G}$ & 5.0 & 1.67 & 22.0 & 0.33 & 4.5 & 1.0 & 8.67 & 0.825 & 0.78 \\
$\mathrm{H}$ & 6.25 & 1.44 & 23.5 & 0.23 & 4.5 & 1.0 & 13.0 & 0.825 & 0.78 \\
$\mathrm{I}$ & 7.5 & 2.50 & 48.0 & 0.33 & 4.5 & 1.0 & 13.0 & 0.825 & 0.78 \\
$\mathrm{~J}$ & 9.0 & 4.31 & 100 & 0.48 & 4.5 & 1.0 & 13.0 & 0.825 & 0.78 \\
$\mathrm{~K}$ & 11.25 & 3.75 & 110 & 0.33 & 4.5 & 1.0 & 19.5 & 0.825 & 0.78 \\
\hline
\end{tabular}


identified on the conventional regime diagram [39]. There have been several previous analyses by other authors [53-55] where flamelet modelling was carried out for $L e<1$ flames for small-scale physics (e.g. FSD and SDR closures) even when the large scale equilibrium between flame surface and turbulent motion was not achieved due to thermo-diffusive instabilities, and the same approach has been adopted here. It was discussed in Ref. [9] that the turbulent flame speed statistics in the non-unity Lewis number cases B-E and G are consistent with previous experimental observations [55]. A model should be robust enough to address the effects of $L e, \tau$ and $\mathrm{Re}_{t}$, and especially LES models should be able to handle unsteady cases and thus the cases A-K have been considered for the current analysis. It was shown in Ref. [38] that the leading order contributors in the SDR transport remain in approximate equilibrium even for $L e<1$ flames (where the effects of thermo-diffusive instability are strong), which is the assumption based on which Eq. $5 \mathrm{i}$ was proposed.

The DNS datasets have been explicitly filtered using the integral $\overline{Q(\vec{x})}=$ $\int Q(\vec{x}-\vec{r}) G(\vec{r}) d \vec{r}$ for $\Delta$ ranging from $\Delta \approx 0.4 \delta_{\text {th }}$ to $\Delta \approx 2.8 \delta_{\text {th }}$ where $G(\vec{r})=$ $\left(6 / \pi \Delta^{2}\right)^{3 / 2} \exp \left(-6 \vec{r} \cdot \vec{r} / \Delta^{2}\right)$. This range of filter widths is comparable to the range of $\Delta$ used in several previous a-priori DNS analyses [6, 7, 29, 31, 32, 34, 56], and span a useful range of length scales from $\Delta$ comparable to $0.4 \delta_{t h} \approx 0.71 \delta_{z}\left(\delta_{z}=\alpha_{T 0} / S_{L}\right.$ is the Zel'dovich flame thickness with $\alpha_{T 0}$ being the unburned gas thermal diffusivity) where the flame is partially resolved, up to $2.8 \delta_{t h} \approx 5 \delta_{z}$ where the flame becomes fully unresolved and $\Delta$ is comparable to the integral length scale $l$.

\section{Results \& Discussion}

\subsection{Volume-averaged behaviour}

In order to assess the performances of the SDR models it is useful to compare the predictions of the volume-averaged value of density-weighted SDR (i.e. $\left\langle\bar{\rho} \tilde{N}_{c}\right\rangle_{V}$ ) as this quantity is expected to be proportional to the volume-averaged reaction rate $\langle\overline{\dot{w}}\rangle_{V}$ under the flamelet assumption according to $\overline{\dot{w}}=2 \bar{\rho} \tilde{N}_{c} /\left(2 c_{m}-1\right)$ where $\langle\ldots\rangle_{V}$ indicates a volume-averaging operation. In the context of FSD based closure a wrinkling factor $\Xi_{F}^{V}$ is often defined based on volume-averaged quantities in the following manner $\Xi_{F}^{V}=\left\langle\Sigma_{g e n}\right\rangle_{V} /\langle|\nabla \bar{c}|\rangle_{V}$ to assess the validity of the power-law closure for FSD (i.e. $\left.\Sigma_{g e n}=|\nabla \bar{c}|\left(\Delta / \eta_{i}\right)^{D_{f}-2}\right)[29-32,34]$. Dunstan et al. [6] defined a SDR based wrinkling factor $\Xi_{D}$ drawing on the analogy with $\Sigma_{g e n}=|\nabla \bar{c}|\left(\Delta / \eta_{i}\right)^{D_{f}-2}$, as follows:

$$
\Xi_{D}=\frac{\tilde{N}_{c}}{\tilde{D} \nabla \tilde{c} \cdot \nabla \tilde{c}}
$$

Dunstan et al. [6] also explored the possibility of modelling $\Xi_{D}$ by using a power-law given by Eq. 1 by drawing analogy with the power-law based FSD closure. The ratio of the total volume-averaged density-weighted SDR (i.e. $\left\langle\bar{\rho} \tilde{N}_{c}\right\rangle_{V}$ ) and its resolved component (i.e. $\langle\bar{\rho} \tilde{D} \nabla \tilde{c} . \nabla \tilde{c}\rangle_{V}$ ) can be considered as the measure of global wrinkling factor in the context of SDR based closure, which can be defined as $[6,7]$ :

$$
\Xi_{D}^{V}=\frac{\left\langle\bar{\rho} \tilde{N}_{c}\right\rangle_{V}}{\langle\bar{\rho} \tilde{D} \nabla \tilde{c} . \nabla \tilde{c}\rangle_{V}}
$$


This global SDR-based wrinkling factor is defined in terms of $\left\langle\bar{\rho} \tilde{N}_{c}\right\rangle_{V}$ and not as a function of $\left\langle\tilde{N}_{c}\right\rangle_{V}$ because $\left\langle\bar{\rho} \tilde{N}_{c}\right\rangle_{V}$ remains proportional to $\langle\overline{\dot{w}}\rangle_{V}$ according to $\overline{\dot{w}}=2 \bar{\rho} \tilde{N}_{c} /\left(2 c_{m}-\right.$ 1) under the flamelet assumption because $c_{m}$ is a constant for a given thermo-chemistry. Here the expression given by Eq. 8 has been used to assess the validity of power-law closure (i.e. Eq. 1) and the fidelity of the predictions of the volume-averaged behaviour of $\left\langle\bar{\rho} \tilde{N}_{c}\right\rangle_{V}$ by different SDR closures (Eqs. 1 and 5i) considered in this analysis.

The variation of $\Xi_{D}^{V}$ with $\Delta / \delta_{t h}$ for cases A-G and K are shown in Fig. 1 on a log$\log$ plot. The cases $\mathrm{H}, \mathrm{I}$ and $\mathrm{J}$ are qualitatively similar to cases $\mathrm{G}, \mathrm{E}$ and $\mathrm{K}$ respectively and thus are not shown in Fig. 1 and subsequent figures. A linear variation of $\log \left(\Xi_{D}^{V}\right)$ with $\log \left(\Delta / \delta_{t h}\right)$ indicates a power-law dependence between $\Xi_{D}^{V}$ and $\Delta$ (see Eq. 1), which can be seen in Fig. 1 for $\Delta>\delta_{t h}$ but not for $\Delta<\delta_{t h}$. Although the linear variation
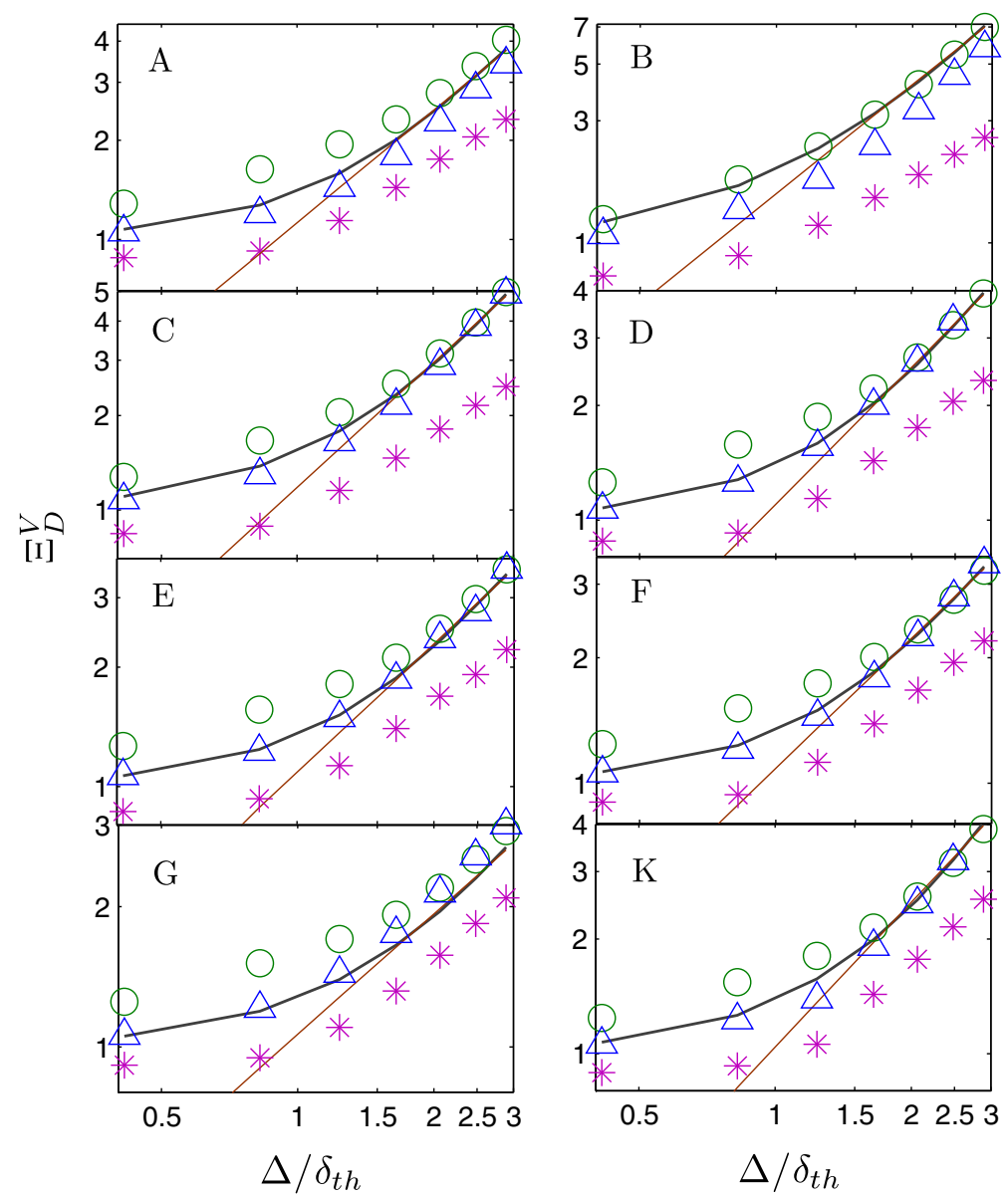

Fig. 1 Variations of $\Xi_{D}^{V}$ (ـ) with $\Delta / \delta_{t h}$ on a log-log plot along with the predictions of Power-law model (i.e. Eq. 1) (o) with dynamic $\alpha_{D}$, static LES-G model (Eq. 5i with $\beta_{c}$ according to Eq. 5iv) (*⿻) and dynamic LES-G model (Eq. 5i with $\beta_{c}$ according to Eq. 7) ( $\Delta$ ) in cases A-G and K. The linear region describing the power-law given by Eq. 1 is marked by the solid line following least-squares fit corresponding to the largest slope 
is seen for $\Delta / \delta_{t h}$ ranging from 1.25 to 3 , this range is not too small because it is quite common to observe this normalised filter width to be much smaller than unity in many recent LES studies. It would be ideal to have large values for this normalised filter width in DNS analysis but at $\Delta \approx 3.0 \delta_{t h}$ the filter width becomes comparable to the integral length scale $l$. The dynamic modelling of both Eqs. 3 and $5 \mathrm{i}$ is dependent on the identity $\overbrace{\rho N_{c}}=\overbrace{\bar{\rho} \tilde{N}_{c}}$, which is independent of the filter size. Thus, the dynamic model predictions are unlikely to be affected for large filter widths for which both the basic filter and test filter sizes become comparable to the integral length scale (e.g. $\Delta=2.8 \delta_{t h} \approx 5 \delta_{z} \approx$ $l$ for cases A-F and I).

The slope of the best-fit straight line with the steepest slope in Fig. 1 provides a global value of $\alpha_{D}$ and the intersection of this line with the $\Xi_{D}^{V}=1.0$ (or $\log \Xi_{D}^{V}=0.0$ ) gives the measure of $\eta_{i D} / \delta_{t h}$. The values of $\alpha_{D}$ and $\eta_{i D} / \delta_{t h}$ for all cases considered here can be found in Ref. [7] but are repeated here in Table 2 for the sake of subsequent discussion in this paper. It can be seen from Table 2 that $\alpha_{D}$ assumes higher values for cases with smaller value of $L e$ for a given value of $u^{\prime} / S_{L}$, whereas $\alpha_{D}$ increases with increasing $u^{\prime} / S_{L} \sim$ $R e_{t}^{1 / 4} K a^{1 / 2} \sim R e_{t}^{1 / 2} / D a^{1 / 2}$ for a given value of $L e$. By contrast, $\eta_{i D}$ remains of the order of $\delta_{t h}$ for all cases. Table 2 shows that $\alpha_{D}<1.0$ for weakly turbulent flames (e.g. cases $\mathrm{G}$ and $\mathrm{H}$ ) which also leads to $\Xi_{D}^{V}>1.0$ for $\Delta>\delta_{t h}$. An increase of $\alpha_{D}$ with decreasing $L e$ for a given value of $L e$ suggests an increase in the extent of flame wrinkling, which can be substantiated from the values of normalised flame surface area $A_{T} / A_{L}$, which is provided in Table 2 where the flame surface area has been evaluated using the volume integral $A=\int_{V}|\nabla c| d V$ with the superscripts ' $T$ ' and ' $L$ ' referring to turbulent and laminar flame quantities respectively. Table 2 further shows that flame area generation increases with increasing $u^{\prime} / S_{L}$ for a given value of $L e$, which in turn gives rise to an increasing trend of $\alpha_{D}$ with an increase in $u^{\prime} / S_{L}$. It has been demonstrated in Refs. [6, 7] that the power-law model (i.e. Eq. 1) does not adequately predict the local behaviour of $\tilde{N}_{c}$ even when $\alpha_{D}$ and $\eta_{i D}$ obtained from DNS data in Table 2 are used. Interested readers are referred to Refs. [6, 7] for more discussion on the performance of the static version of the power-law model.

The prediction of the LES-G model (i.e. Eq. 5i) with $\beta_{c}$ given by Eq. 5 iv is also shown in Fig. 1, which shows that Eq. 5i satisfactorily predicts the variation of $\Xi_{D}^{V}$ with $\Delta$ but this is expected as the parameterisation given by Eq. $5 \mathrm{iv}$ is designed to capture the magnitude

Table 2 List of $\alpha_{D}, \eta_{i D} / \delta_{t h}$ and $A_{T} / A_{L}$ for all cases

\begin{tabular}{llll}
\hline Case & $\alpha_{D}$ & $\eta_{i D} / \delta_{t h}$ & $A_{T} / A_{L}$ \\
\hline $\mathrm{A}$ & 1.13 & 0.90 & 1.94 \\
$\mathrm{~B}$ & 1.42 & 0.73 & 3.93 \\
$\mathrm{C}$ & 1.32 & 0.88 & 2.66 \\
$\mathrm{D}$ & 1.19 & 0.92 & 2.11 \\
$\mathrm{E}$ & 1.07 & 0.93 & 1.84 \\
$\mathrm{~F}$ & 1.04 & 0.93 & 1.76 \\
$\mathrm{G}$ & 0.86 & 0.93 & 1.1 \\
$\mathrm{H}$ & 0.88 & 0.94 & 1.25 \\
$\mathrm{I}$ & 1.11 & 0.98 & 1.85 \\
$\mathrm{~J}$ & 1.18 & 0.96 & 3.75 \\
$\mathrm{~K}$ & 1.26 & 0.97 & 3.80 \\
\hline
\end{tabular}


of $\left\langle\bar{\rho} \tilde{N}_{c}\right\rangle_{V}$ [7]. However, Fig. 1 suggests that an accurate estimation of $\tilde{N}_{c}$ can be obtained using Eq. $5 \mathrm{i}$ and the empiricism involved in $\beta_{c}$ parameterisation (i.e. similar to Eq. $5 \mathrm{i}$ ) can be avoided if $\beta_{c}$ can be evaluated using Eq. 7 according to the dynamic formulation.

The predictions of Eq. 1 with dynamic evaluation of $\alpha_{D}$ according to Eq. 4 are compared to the mean value of $\tilde{N}_{c}$ conditional on $\tilde{c}$ obtained from DNS in Fig. 2 for cases A-G and $\mathrm{K}$ at $\Delta \approx 0.4 \delta_{t h}$ and $\Delta \approx 2.8 \delta_{t h}{ }^{3}$ The volume-averaging involved in dynamic evaluation of $\alpha_{D}$ (see Eq. 4) is carried out by ensemble averaging the relevant quantities of using $(2 n)^{3}$ cells around a given grid point, and it was found that results did not change significantly for $10>n>3$. Here the results are shown for $n=4$. The same procedure was used for volume-averaging process involved in the dynamic evaluation of $\beta_{c}$ using Eq. 7. The prediction of the mean value of $\tilde{N}_{c}$ conditional on $\tilde{c}$ by the dynamic version of the power-law (i.e. Eq. 1) model deteriorates (significant under-prediction for $\Delta<\delta_{t h}$, and over-prediction for $\Delta>\delta_{t h}$ ) when the averaging is performed over the whole computational domain for the evaluation of $\alpha_{D}$ (using Eq. 4). The prediction of the mean value of $\tilde{N}_{c}$ conditional on $\tilde{c}$ by the dynamic version of the SDR-G model approaches that of the static version of this model (i.e. $\beta_{c}$ according to Eq. 5iv) when the averaging is performed over the whole computational domain for the evaluation of $\beta_{c}$ (using Eq. 7). However, this averaging over the whole domain while calculating $\alpha_{D}$ and $\beta_{c}$ using Eqs. 4 and 7 respectively ensures that the volume-averaged value of density-weighted SDR is satisfactorily predicted $(\because$ $<\overbrace{\overline{\rho N_{c}}}>_{V}=\overbrace{\left\langle\bar{\rho} \tilde{N}_{c}\right\rangle_{V}})$.

Note that $\alpha_{D}$ is a three-dimensional variable in the context of dynamic modelling and thus it is ensemble averaged conditional on bins of $\tilde{c}$ in Fig. 3. ${ }^{4}$ The variations of the mean values of $\alpha_{D}$ conditional on $\tilde{c}$ for cases $\mathrm{A}-\mathrm{G}$ and $\mathrm{K}$ at $\Delta \approx 0.4 \delta_{t h}$ and $\Delta \approx 2.8 \delta_{\text {th }}$ are shown in Fig. 3. It is clear from Fig. 3 that dynamic formulation according to Eq. 4 successfully captures the increase in power-law exponent $\alpha_{D}$ with increasing $R e_{t}$ for a given value of $L e$. Moreover, it can be seen from Fig. 3 that $\alpha_{D}$ increases with decreasing Le. Figure 3 demonstrates that $\alpha_{D}$ according to Eq. 4 shows considerable local variation of power-law exponent within the flame brush for $\Delta>\delta_{t h}$ (e.g. $\Delta \approx 2.8 \delta_{t h}$ ). Moreover, Fig. 3 shows that the dynamic formulation shows a reduction in $\alpha_{D}$ with decreasing $\Delta$. It can be seen from Fig. 2 that the dynamic power-law model prediction under-predicts the mean value of $\tilde{N}_{c}$ conditional on $\tilde{c}$ towards the unburnt side of the flame brush for $\Delta>\delta_{t h}$ (e.g. $\Delta \approx 2.8 \delta_{t h}$ ) for all cases, and the qualitative variation of $\tilde{N}_{c}$ with $\tilde{c}$ is not captured by the dynamic model for $L e<<1$ cases (e.g. cases B and C). However, the variation of mean value of $\tilde{N}_{c}$ conditional on $\tilde{c}$ is satisfactorily captured for $\Delta<\delta_{t h}$ (e.g. $\Delta \approx 0.4 \delta_{t h}$ ) for $L e \approx 1$ cases but the dynamic model under-predicts the mean value of $\tilde{N}_{c}$ conditional on $\tilde{c}$ for $L e<<1$ cases (e.g. cases B and C) even at small filter widths (i.e. $\Delta<\delta_{t h}$, for example $\Delta \approx 0.4 \delta_{t h}$ ). The predictions of $\Xi_{D}^{V}$ according to Eq. 1 with dynamic $\alpha_{D}$ (i.e. Eq. 4) evaluation are also shown in Fig. 1, which shows that dynamic evaluation of $\alpha_{D}$ results in the under-prediction of $\Xi_{D}^{V}$ with increasing $\Delta$, and this tendency increases with decreasing $L e$ and is particularly prevalent for flames with $L e<<1$ (e.g. cases B and C).

\footnotetext{
${ }^{3}$ The width of the power-law range and the actual value of power-law coefficient do not play important roles in the results shown in Fig. 2. For dynamic power-law model the exponent $\alpha_{D}$ is calculated locally. It was shown in Ref. [7] that the static power-law expression fails to predict the local behaviour of $\tilde{N}_{c}$ for the values of $\alpha_{D}$ and $\eta_{i D}$ for which $<\bar{\rho} \tilde{N}_{c}>_{D}$ is accurately predicted.

${ }^{4} \mathrm{~A}$ similar method is used to ensemble average the dynamically evaluated $\beta_{c}$ conditional on bins of $\tilde{c}$, which is shown later in Fig. 5.
} 

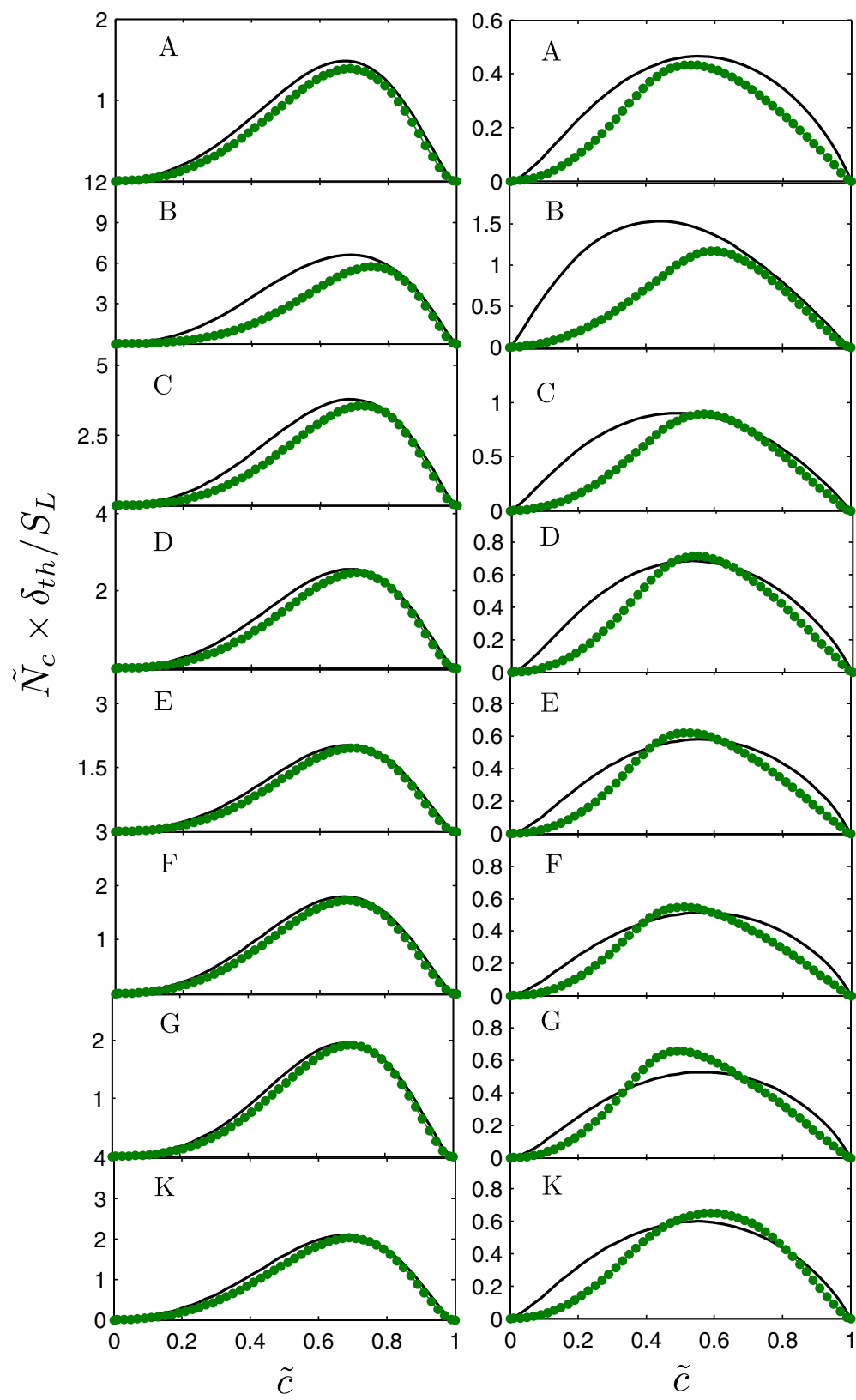

Fig. 2 Variations of $\tilde{N}_{c} \times \delta_{t h} / S_{L}$ (—) conditionally averaged in bins of $\tilde{c}$ along with the predictions of power-law model ( $\longrightarrow$ ) with dynamic evaluation of $\alpha_{D}$ for $\Delta \approx 0.4 \delta_{t h}$ (left column) and $\Delta \approx 2.8 \delta_{t h}$ (right column) in cases A-G and $\mathrm{K}$

It is possible propose an alternative power-law model in the following manner:

$$
\overline{\rho N_{c}}=\bar{\rho} \tilde{N}_{c}=\bar{\rho} \tilde{D} \nabla \tilde{c} . \nabla \tilde{c}\left(1+\frac{\Delta}{\delta_{t h}}\right)^{\alpha_{D}^{\prime}}
$$



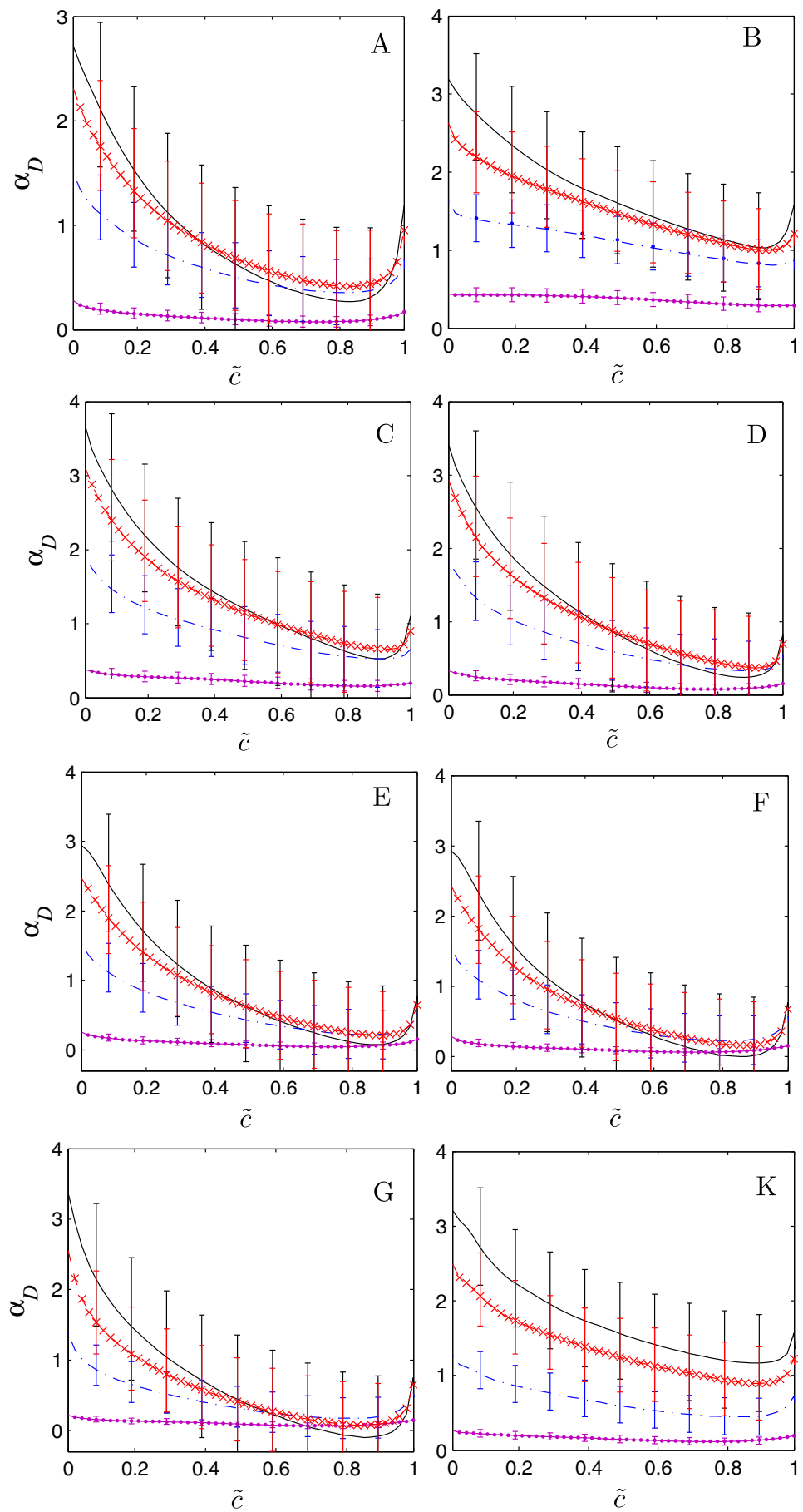

Fig. 3 Variations of dynamically evaluated $\alpha_{D}$ (according to Eq. 4) conditionally averaged in bins of $\tilde{c}$ for $\Delta \approx 0.4 \delta_{t h}$ ( $), \Delta \approx 1.2 \delta_{t h}(\longrightarrow), \Delta \approx 2.0 \delta_{t h}\left(\longrightarrow\right.$ ) and $\Delta \approx 2.8 \delta_{t h}$ ) with the bars indicating one standard deviation variation over the mean in cases $A-G$ and $K$ 
which leads to the following expression under the assumption of scale-similarity:

$$
\begin{aligned}
\overbrace{\bar{\rho} N_{c}} & =\overbrace{\bar{\rho} \tilde{N}_{c}}=\overbrace{\left(\overline{\bar{\rho} \tilde{D} \nabla \tilde{c} . \nabla \tilde{c})} \cdot\left(1+\Delta / \delta_{t h}\right)^{\alpha_{D}^{\prime}}\right.} \\
& =\overbrace{\bar{\rho}} \overbrace{\tilde{D}} \nabla \overbrace{\tilde{c}} \cdot \nabla \overbrace{\tilde{c}} \cdot(1+\overbrace{\bar{\Delta}} / \delta_{t h})^{\alpha_{D}^{\prime}}
\end{aligned}
$$

Equation 9ii provides:

$$
\alpha_{D}^{\prime}=\frac{\ln [<\overbrace{(\bar{\rho} \tilde{D} \nabla \tilde{c} \cdot \nabla \tilde{c})}>_{D} /<\overbrace{\bar{\rho}} \overbrace{\tilde{D}} \nabla \overbrace{\tilde{c}} \cdot \nabla \overbrace{\tilde{c}}>_{D}]}{\ln \left[(1+\overbrace{\bar{\Delta}} / \delta_{t h}) /\left(1+\Delta / \delta_{t h}\right)\right]}
$$

However, the performance of Eq. 9i remains inferior than the dynamic version of the model given by Eq. 1 and thus are not discussed here.

The applicability of scale-similarity for quantities related to scalar gradient (e.g. SDR and Flame FSD) is debatable but the assumption of scale-similarity was successfully used in the past for the closure of FSD [29-31, 34]. However, the results in Figs. 1 and 2 suggest that the strong assumption regarding scale independent functional form of the closure (i.e. $\alpha_{D}$ does not change between actual and test filter scales) that has been invoked while deriving Eq. 4 may not be strictly valid, as SDR for passive scalars is known to exhibit multi-fractal nature $[57,58,60,61]$ and a similar behaviour is likely to be present also for reacting flows. Thus, a single power-law exponent may not be suitable to describe the statistical behaviour of $\tilde{N}_{c}$. Thus, the inaccuracies associated with the assumption of scale independent functional form of the closure while deriving Eq. 4 might have strong implications for highly wrinkled flames with $L e<<1$ (e.g. case B), which leads to a discrepancy between the predictions of local and volume-integrated behaviours of SDR according to the dynamic power-law model.

The predictions of the LES-G model (i.e. Eq. 5i) with dynamic evaluation of $\beta_{c}$ (according to Eq. 7) are compared to the same model prediction with static $\beta_{c}$ (according to Eq. 5iv) and mean value of $\tilde{N}_{c}$ conditional on $\tilde{c}$ obtained from DNS in Fig. 4 for cases A-G and K at $\Delta \approx 0.4 \delta_{t h}$ and $\Delta \approx 2.8 \delta_{t h}$. The variations of the mean values of dynamically evaluated $\beta_{c}$ conditional on $\tilde{c}$ for cases $\mathrm{A}-\mathrm{G}$ and $\mathrm{K}$ at $\Delta \approx 0.4 \delta_{t h}$ and $\Delta \approx 2.8 \delta_{t h}$ are shown in Fig. 5 along with the standard deivation values with respect to the conditional mean. The predictions of $\Xi_{D}^{V}$ according to Eq. 5i with dynamic $\beta_{c}$ evaluation are also shown in Fig. 1. It is evident from Fig. 5 that Eq. 7 predicts appreciable local variation of $\beta_{c}$ within the flame brush and the mean value of $\beta_{c}$ remains greater than $2 /\left(2 c_{m}-1\right)$ for all cases $\left(2 /\left(2 c_{m}-1\right)\right.$ remains close to 3.0 for the $c_{m}$ values given in Table 1$)$ for $\Delta>>\delta_{t h}$ (e.g. $\left.\Delta=2.8 \delta_{t h}\right)$. Moreover, the standard deviation values of dynamically evaluated $\beta_{c}$ suggest a considerable probability of finding $\beta_{c}>2 /\left(2 c_{m}-1\right)$. The pdfs of $\beta_{c}$ using the samples corresponding to $0.05 \leq \tilde{c} \leq 0.95$ for cases $\mathrm{H}$ and $\mathrm{K}$ at $\Delta=2.8 \delta_{t h}$ are shown in Figs. $6 \mathrm{a}$ and $\mathrm{b}$ respectively, which indicate that $\beta_{c}$ values are not significantly limited by the realisability condition $2 /\left(2 c_{m}-1\right)$ for $\Delta>>\delta_{t h}$ (e.g. $\left.\Delta=2.8 \delta_{t h}\right)$. For small values of $\Delta$ (e.g. $\left.\Delta=0.4 \delta_{t h}\right) \quad \beta_{c}$ values are limited by the realisability condition $2 /\left(2 c_{m}-1\right)$ and the pdf of $\beta_{c}$ peaks around $2 /\left(2 c_{m}-1\right)$ (not shown here). The shape of pdf and the aforementioned findings do not change if the samples corresponding to $0.1 \leq \tilde{c} \leq 0.9$ are used instead of the samples with $0.05 \leq \tilde{c} \leq 0.95$. The pdfs $\beta_{c}$ for other cases are qualitative similar to those in cases $\mathrm{H}$ and $\mathrm{K}$, and thus are not shown here.

It has been demonstrated in Refs. $[18,24,25]$ that the parameter $\beta_{c}$ is closely linked with the modelling of the terms $-\overline{2 D \nabla \cdot\left(\rho S_{d} \vec{N}|\nabla c|\right)|\nabla c|}+2 \overline{\rho D S_{d} \nabla \cdot \vec{N}|\nabla c|^{2}}$ which depend on the correlation between displacement speed $S_{d}=(D c / D t) /|\nabla c|$ and curvature $\kappa_{m}=\nabla \cdot \vec{N} / 2=\nabla \cdot(-\nabla c /|\nabla c|) / 2$, similar to the curvature term (i.e $\overline{2 S_{d} \kappa_{m}|\nabla c|}$ ) in the 

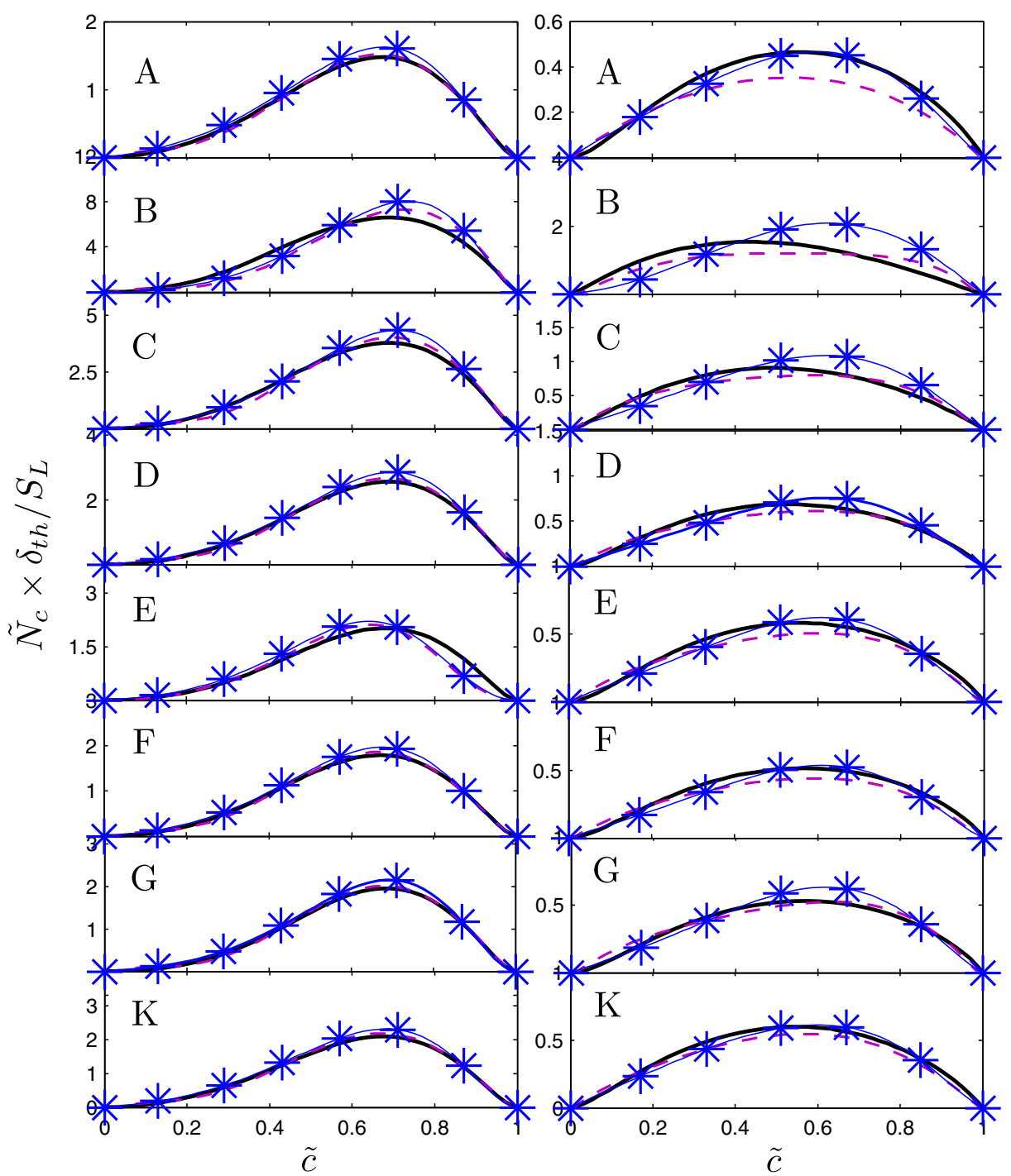

Fig. 4 Variations of $\tilde{N}_{c} \times \delta_{t h} / S_{L}$

) conditionally averaged in bins of $\tilde{c}$ along with the predictions of static LES-G model (Eq. 5i with $\beta_{c}$ according to Eq. 5iv) (——) and dynamic LES-G model (Eq. 5i with $\beta_{c}$ according to Eq. 7 ) (_ _ _ - ) for $\Delta \approx 0.4 \delta_{t h}$ (left column) and $\Delta \approx 2.8 \delta_{t h}$ (right column) in cases A-G and $\mathrm{K}$

FSD $\Sigma_{g e n}=\overline{|\nabla c|}$ transport equation [54, 56]. It is well-known that the surface averaged curvature and the FSD curvature term exhibit significant differences in behaviour in different regions of the flame brush $[9,51,53,54,56]$ and by the same token the parameter $\beta_{c}$ shows significant variation of within the flame brush.

Figure 5 indicates an increasing trend of $\beta_{c}$ with increasing $\tau$, as suggested by the empirical parameterization given by Eq. 5iv. It is evident from Fig. 4 that the LES-G model with dynamic evaluation of $\beta_{c}$ captures the behaviour of mean value of $\tilde{N}_{c}$ conditional on $\tilde{c}$ obtained from DNS data either comparably or better than the static version of the LES-G 

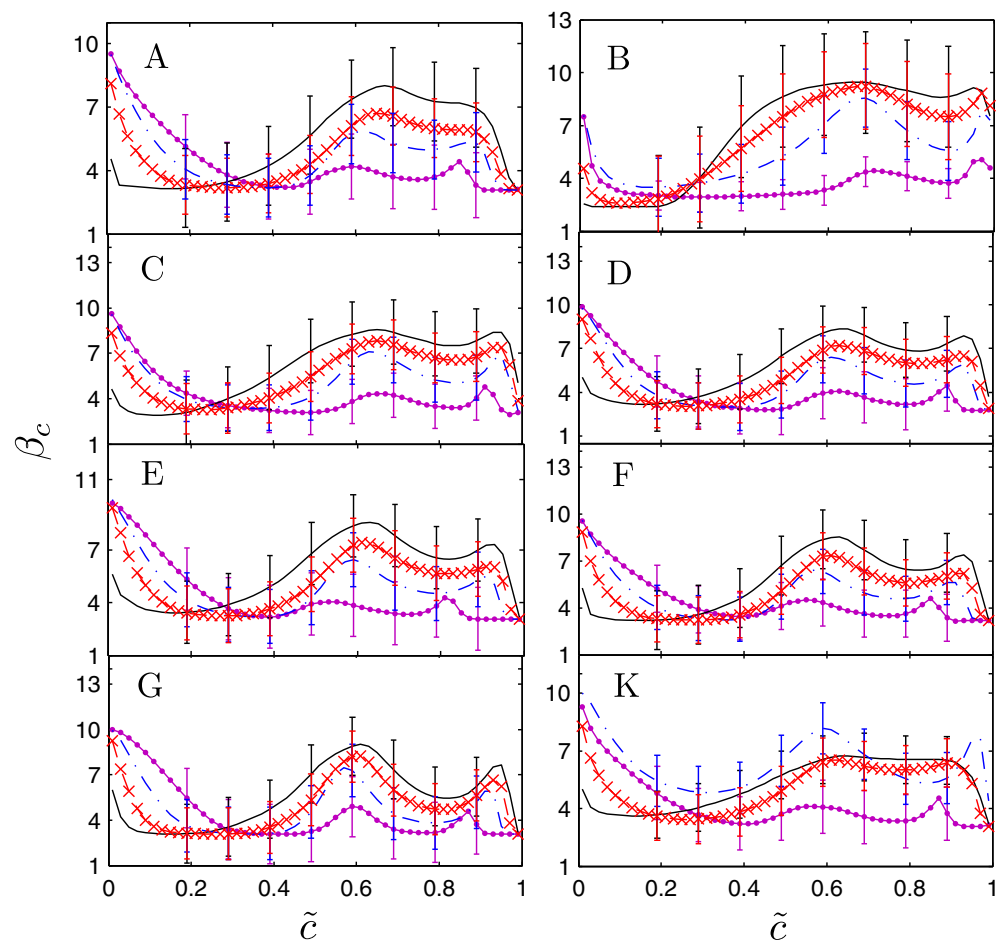

Fig. 5 Variations of dynamically evaluated $\beta_{c}$ conditionally averaged in bins of $\tilde{c}$ for $\Delta \approx 0.4 \delta_{t h}$

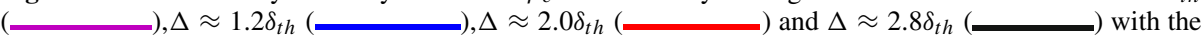
bars indicating one standard deviation variation over the mean in cases A-G and $\mathrm{K}$

model with $\beta_{c}$ parameterisation according to Eq. 5iv. The advantages of dynamic model are particularly prominent for small values of $R e_{t}$ and for flames with small $L e$ (e.g. cases B, $\mathrm{G}$ and $\mathrm{H}$ ) where the dynamic model satisfactorily captures $\tilde{N}_{c}$ variation with $\tilde{c}$, whereas the static version of the model overpredicts the mean value of $\tilde{N}_{c}$ conditional on $\tilde{c}$ for a major portion of the flame brush for $\Delta>>\delta_{t h}$ (i.e. $\Delta \approx 2.8 \delta_{t h}$ ). For the $L e<<1$ cases, the equilibrium between flame surface and turbulent motion is not maintained due to thermodiffusive instabilities and thus it is perhaps not surprising that the static version of the LES-G model (with $\beta_{c}$ parameterisation according to Eq. 5iv) cannot adequately capture the local behaviour of $\tilde{N}_{c}$ within the flame brush. However, the dynamic formulation (i.e. $\beta_{c}$ according to Eq. 7) allows for the inclusion of local flame turbulence interaction and thus the dynamic formulation of LES-G model works satisfactory for the $L e<<1$ cases. Moreover, Fig. 1 suggests that the prediction of $\Xi_{D}^{V}$ according to Eq. 5i with dynamic $\beta_{c}$ evaluation (i.e. Eq. 7) remains satisfactory and comparable to the prediction of the model with static $\beta_{c}$ parameterization (i.e. Eq. 5iv). However, the dynamic version of the LES-G model (i.e. Eq. 5i) does not depend on any empirical parametersation of $\beta_{c}$ similar to Eq. 5 iv but inherently accounts for $R e_{t}, L e$ and $\tau$ dependences of $\tilde{N}_{c}$ for a range of different filter widths $\Delta$. A comparison between Figs. 2 and 4 further reveals that Eq. 5i with dynamic $\beta_{c}$ evaluation is more successful in capturing the local behaviour of $\tilde{N}_{c}$ than the power-law model (i.e. Eq. 1) with dynamic evaluation of $\alpha_{D}$. Moreover, Fig. 1 suggests that $\Xi_{D}^{V}$ according to Eq. $5 \mathrm{i}$ with dynamic $\beta_{c}$ evaluation remains better than the prediction of Eq. 1 with dynamic 

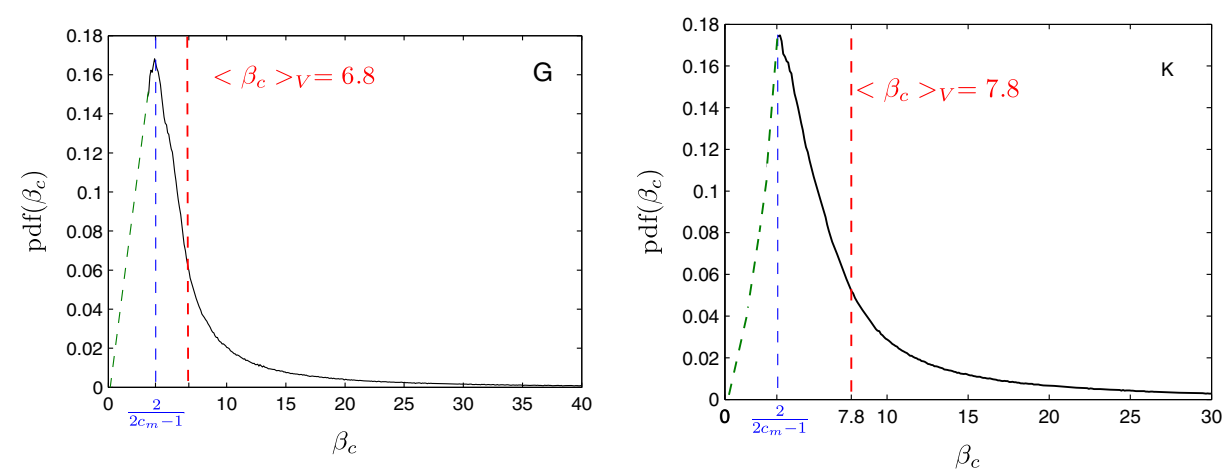

Fig. 6 Pdfs of $\beta_{c}$ for the samples given by $0.05 \leq \tilde{c} \leq 0.95$ at $\Delta \approx 2.8 \delta_{t h}$ for cases (a) $\mathrm{H}$ and (b) $\mathrm{K}$ cases. The realisability limit, $2 /\left(2 c_{m}-1\right)$ is also marked above

evaluation of $\alpha_{D}$. This suggests that the power-law models, though widely used for the purpose of algebraic closure of $\Sigma_{g e n}$ [29-34], may not be suitable for SDR $\tilde{N}_{c}$ modelling and this behaviour perhaps arises due to multi-fractal nature of SDR, which was observed previously for passive scalar mixing [57-61]. By contrast, both static and dynamic versions of the LES-G model (i.e. Eq. 5i) are more successful in predicting SDR accurately than the power-law based models for a range of different values of $R e_{t}, L e$ and $\tau$ (see Figs. 1 and 4).

The assumption of scale independent functional form of the closure is indeed questionable for modelling the quantities related to scalar gradient in premixed flames. However, this concept was successfully used for modelling the generalised FSD $\Sigma_{\text {gen }}$ [29-34] in the past using a power-law approach. However, previous findings [6, 7] and current analysis indicate that the concept of scale independent functional form of the closure may not be suitable for SDR modelling in the context of LES using a power-law approach. Although the assumption of scale independent functional form of the closure is invoked for dynamic evaluation of $\beta_{c}$ but this assumption is applied to the function $f_{1}$ which is dependent on $u_{\Delta}^{\prime} / S_{L}$ and $\Delta / \delta_{t h}$ (see Eqs. 6i and 6ii). As the scale independent functional form of the closure has been demonstrated to be successful in capturing the quantities associated with turbulence (e.g. $u_{\Delta}^{\prime}$ ) [62-64], this assumption works better for dynamic evaluation of $\beta_{c}$ than the dynamic evaluation of power-law exponent.

\section{Conclusions}

In the present analysis dynamic algebraic closures of SDR $\tilde{N}_{c}$ of reaction progress variable $c$ have been assessed by explicitly LES filtering a DNS database of statistically planar turbulent premixed flames with a range of different values of $\tau, L e$ and $R e_{t}$. An algebraic model for SDR which was originally proposed in the context of RANS (i.e. RANS-CS model) has been extended here for the purpose of LES and the possibility of SDR closure using a power-law has also been explored here. The performances of the RANS-extended algebraic SDR closure (i.e. LES-G model) have been assessed with respect to $\tilde{N}_{c}$ extracted from DNS data for the model parameters, which are either dynamically evaluated or calculated by using a presumed empirical parameterisation. The possibility of SDR closure using a power-law model based on dynamic evaluation of the model parameter $\alpha_{D}$ has been assessed with respect to $\tilde{N}_{c}$ extracted from DNS data. This power-law model is found 
to capture the local variation of $\tilde{N}_{c}$ both qualitatively and quantitatively for small filter width for $L e \approx 1$ flames, but under-predicts $\tilde{N}_{c}$ for $L e<<1$ flames. The prediction of volume-averaged SDR also suffers especially for $L e<<1$ flames. The under-predictions of volume-averaged behaviour of density-weighted SDR increase with increasing filter width. A power-law for $\tilde{N}_{c}$ with a single global value of power-law exponent has been found to be inadequate for the modelling of SDR in the context of LES possibly due to the multi-fractal nature of SDR.

A recently proposed SDR model (i.e. LES-G model), extended from an SDR model for RANS, has been shown to capture the local behaviour of SDR better than the power-law model for which the volume-averaged behaviour of SDR is appropriately captured. The empirical parameterisation of $\beta_{c}$ in the LES-G model can be avoided by using a dynamic formulation which captures the local behaviour of SDR either comparably or better than the static formulation for a range of different values of $\Delta, \tau, L e$ and $R e_{t}$, whereas the volumeaveraged SDR is also adequately predicted. Thus, the dynamic formulation based on the LES-G model seems to be a viable option for algebraic $\tilde{N}_{c}$ closure for turbulent premixed combustion. However, this newly proposed model has been assessed here based on simple chemistry DNS for moderate values of $R e_{t}$ with decaying turbulence and thus needs to be assessed further based on detailed chemistry based DNS data for higher values of $R e_{t}$ despite a previous analysis [43] demonstrated that the SDR statistics obtained from detailed chemistry DNS remain qualitatively similar to the conclusions drawn from simple chemistry DNS. Although the static version of the LES-G model has already been implemented in actual LES simulations and satisfactory agreement with experimental findings has been obtained $[8,41]$, the proposed dynamic model also needs to be implemented in actual LES simulations in a configuration for which experimental data is available for the purpose of $a$ posteriori assessment. Furthermore, in combustion LES the sub-grid modelling also affects the resolved scale behaviour, which may interact with flame-turbulence interaction in a complicated manner. Thus, detailed a-posteriori assessment of the dynamic formulations of Eqs. 1 and 5iv will be necessary based on LES simulations before either adopting or rejecting a particular modelling idea because $a$-priori assessment cannot predict beforehand how a particular model will interact with other unclosed terms and the numerical schemes in an actual LES calculation and also there is a possibility that a-priori assessment could be too demanding on the model where the modelling errors do not play an important role in simulation predictions. A-posteriori assessment of the dynamic formulations discussed here will form the basis of future investigations.

Acknowledgments The financial assistance of EPSRC is gratefully acknowledged.

\section{Appendix A: Physical Basis of the Algebraic SDR Model}

The RANS-K model by Kolla et al. [22] is given by:

$$
\breve{\varepsilon}_{c}=\left[2 K_{c}^{*} \frac{S_{L}}{\delta_{t h}}+\left(C_{3}^{\prime}-\tau C_{4}^{\prime} D a_{L}\right) \frac{\breve{\varepsilon}}{\breve{k}}\right] \frac{\breve{c}(1-\breve{c})}{\beta_{1}^{\prime}}
$$

The model parameters in Eq. A1 are given by:

$$
C_{3}^{\prime}=\frac{1.5 \sqrt{K a_{L}}}{\left(1+\sqrt{K a_{L}}\right)} ; \quad C_{4}^{\prime}=\frac{1.1}{\left(1+K a_{L}\right)^{0.4}} \text { and } \beta_{1}^{\prime}=6.7
$$


where $\breve{k}=<\rho u_{i}^{\prime \prime} u_{i}^{\prime \prime}>/<\rho>, \breve{\varepsilon}=<\mu\left(\partial u_{i}^{\prime \prime} / \partial x_{j}\right)\left(\partial u_{i}^{\prime \prime} / \partial x_{j}\right)>/<\rho>$ and $D a_{L}=$ $\breve{k} S_{L} / \breve{\varepsilon} \delta_{t h}$ are the turbulent kinetic energy, its dissipation rate and local Damköhler number respectively, whereas $K a_{L}=\left(\breve{\varepsilon} \delta_{t h}\right)^{1 / 2} S_{L}^{-3 / 2}$ is the local Karlovitz number. Equation A1 is derived based on the equilibrium of the leading order terms in the $\breve{\varepsilon}$ transport equation (i.e. $T_{I}+T_{I I}+T_{I I I}+T_{I V} \approx 0.0$ ) where the terms $T_{I}, T_{I I}, T_{I I I}$ and $T_{I V}$ are given by [22]:

$$
\begin{gathered}
T_{I}=-\left\langle 2 D \frac{[\dot{w}+\nabla \cdot(\rho D \nabla c)]}{\rho} \frac{\partial c}{\partial x_{k}} \frac{\partial \rho}{\partial x_{k}}\right\rangle \\
+2 \frac{\langle D\rangle}{\langle\rho\rangle} \frac{\partial \breve{c}}{\partial x_{k}} \frac{\partial\langle\rho\rangle}{\partial x_{k}}\left[\langle\dot{w}+\nabla \cdot(\rho D \nabla c)\rangle-\frac{\partial\left\langle\rho u_{j}^{\prime \prime} c^{\prime \prime}\right\rangle}{\partial x_{j}}\right] \\
T_{I I}=-2\left\langle\rho D \frac{\partial c^{\prime \prime}}{\partial x_{i}} \frac{\partial u_{i}^{\prime \prime}}{\partial x_{j}} \frac{\partial c^{\prime \prime}}{\partial x_{j}}\right\rangle \\
T_{I I I}=\left\langle 2 D \frac{\partial \dot{w}}{\partial x_{k}} \frac{\partial c}{\partial x_{k}}\right\rangle-2\langle D\rangle \frac{\partial\langle\dot{w}\rangle}{\partial x_{k}} \frac{\partial \breve{c}}{\partial x_{k}} \\
T_{I V}=-2\left\langle\rho D^{2} \frac{\partial^{2} c^{\prime \prime}}{\partial x_{i} \partial x_{j}} \frac{\partial^{2} c^{\prime \prime}}{\partial x_{i} \partial x_{j}}\right\rangle
\end{gathered}
$$

For low Mach number, globally adiabatic, unity Lewis number flames $T_{I}$ can be simplified as: $T_{I} \approx<2 \rho \nabla \cdot \vec{u} \varepsilon_{c}>[18,22]$ so this term can be modelled as: $T_{I}=2 K_{c}^{*}\left(S_{L} / \delta_{t h}\right)<$ $\rho>\breve{\varepsilon}_{c}$ using the definition of $K_{c}^{*}$ (see Eq. 5ii). The terms $<\rho>\left(C_{3}^{\prime}-\tau C_{4}^{\prime} D a_{L}\right)(\breve{\varepsilon} / \breve{k}) \breve{\varepsilon}_{c}$ and $-<\rho>\beta_{1}^{\prime} \breve{\varepsilon}_{c}^{2} /[\breve{c}(1-\breve{c})]$ are the modelled expressions for $T_{I I}$ and $\left(T_{I I I}+T_{I V}\right)$ respectively [18, 22, 24]. The term $<\rho>C_{3}^{\prime}(\breve{\varepsilon} / \breve{k}) \breve{\varepsilon}_{c}$ addresses the generation of SDR arising from the term $T_{I I}$ due to the alignment of $\nabla c$ with the most compressive principal strain rate, whereas $-<\rho>\tau C_{4}^{\prime} D a_{L}(\breve{\varepsilon} / \breve{k}) \breve{\varepsilon}_{c}$ models the destruction of SDR due to the alignment of $\nabla c$ with the most extensive principal strain rate under the action of flame normal acceleration [18, 22, 24]. The effects of flame normal acceleration weaken with increasing Karlovitz number which is accounted for by $K a_{L}$ dependence of $C_{4}^{\prime}$ in Eq. A2. It is demonstrated by Kolla et al. [22] that it is possible to predict turbulent flame speed $S_{T}=2 \sqrt{\left(D_{t} / \rho_{0}\right)(\partial<\dot{w}>/ \partial \breve{c})_{\breve{c}=0}}$ (according to Kolmogorov-Petrovsky-Piskunov (KPP) theorem) accurately for a number of different experimental configurations using $<\dot{w}>=2<\rho>\breve{\varepsilon}_{c} /\left(2 c_{m}-1\right)$ and Eq. A1.

Chakraborty and Swaminathan [10] modified the RANS-K model [22] for non-unity Lewis number flames:

$$
\breve{\varepsilon}_{c}=\left[2 K_{c}^{*} \frac{S_{L}}{L e^{1.88} \delta_{t h}}+\left(C_{3}^{\prime *}-\tau C_{4}^{* *} D a_{L}\right) \frac{\breve{\varepsilon}}{\breve{k}}\right] \frac{\breve{c}(1-\breve{c})}{\beta_{c}^{\prime}}
$$

where

$$
\begin{aligned}
C_{3}^{*} & =\frac{2.0 \sqrt{K a_{L}}}{\left(1+\sqrt{K a_{L}}\right)}, C_{4}^{*}=\frac{1.2(1.0-c)^{b}}{L e^{2.57}\left(1+K a_{L}\right)^{0.4}}, \\
b & =0.2+1.5|1.0-L e| \text { and } \beta_{1}^{\prime}=6.7
\end{aligned}
$$

Equation A7 (i.e. RANS-CS model) is similar to Eq. A1 but the strengthening of density gradient magnitude and flame normal acceleration are addressed by $2 K_{c}^{*} S_{L} /\left(L e^{1.88} \delta_{t h}\right)$ 
and $L e$ dependence of $C_{4}^{*}$ respectively [10, 24]. Equations A7 and A1 offer comparable predictions for $L e=1.0$ flames [10]. A comparison between Eqs. 5iii and A8 reveals that the expressions for $C_{3}^{*}$ and $C_{4}^{*}$ are directly extended from $C_{3}^{\prime *}$ and $C_{4}^{\prime *}$ respectively [7], and the value of $\beta_{c}$ has been modified in comparison to $\beta_{c}^{\prime}$ for the purpose of extending Eq. A7 for LES.

The transport equation of $\tilde{N}_{c}$ takes the following form [7, 38]:

$$
\begin{aligned}
\frac{\partial\left(\bar{\rho} \tilde{N}_{c}\right)}{\partial t} & +\frac{\partial\left(\bar{\rho} \tilde{u}_{j} \tilde{N}_{c}\right)}{\partial x_{j}}=\overline{\frac{\partial}{\partial x_{j}}\left(\rho D \frac{\partial N_{c}}{\partial x_{j}}\right)} \\
& -\frac{\partial\left[\overline{\rho u_{j} N_{c}}-\bar{\rho} \tilde{u}_{j} \tilde{N}_{c}\right]}{\partial x_{j}}+T_{1}+T_{2}+T_{3}+T_{4}+f(D)
\end{aligned}
$$

where $f(D)$ are the terms arising from diffusivity gradients and the terms $T_{1}, T_{2}, T_{3}$ and $T_{4}$ are given by $[7,38]$ :

$$
\begin{gathered}
T_{1}=-2 \frac{D}{\rho}\left[\dot{w}+\frac{\partial}{\partial x_{i}}\left(\rho D \frac{\partial c}{\partial x_{i}}\right)\right] \frac{\partial c}{\partial x_{j}} \frac{\partial \rho}{\partial x_{j}} \\
T_{2}=-2 \overline{\frac{D}{\partial x_{i}} \frac{\partial u_{i}}{\partial x_{j}} \frac{\partial c}{\partial x_{j}}} \\
T_{3}=2 \overline{2 D \frac{\partial \dot{w}}{\partial x_{i}} \frac{\partial c}{\partial x_{i}}} \\
T_{4}=-2 \rho D^{2} \frac{\partial^{2} c}{\partial x_{i} \partial x_{j}} \frac{\partial^{2} c}{\partial x_{i} \partial x_{j}}
\end{gathered}
$$

A recent analysis by Gao et al. [38] has demonstrated that the terms $T_{1}, T_{2}, T_{3}$ and $T_{4}$ behave similarly to the terms $T_{I}, T_{I I}, T_{I I I}$ and $T_{I V}$ respectively for $\Delta>\delta_{t h}$. Thus, the RANS model given by Eq. A7 can be extended to LES, subject to the leading order balance of the terms in the SDR $\tilde{N}_{c}$ transport equation, by drawing analogy to the modelling of unclosed terms of the transport equation of $\breve{\varepsilon}_{c}$ in the context of RANS.

\section{Appendix B: Non-dimensional Conservation Equations}

The non-dimensional mass, momentum, energy and progress variable transport equations are presented below:

$$
\begin{gathered}
\frac{\partial \rho^{+}}{\partial t^{+}}+\frac{\partial\left(\rho^{+} u_{i}^{+}\right)}{\partial x_{i}^{+}}=0 \\
\frac{\partial\left(\rho^{+} u_{i}^{+}\right)}{\partial t^{+}}+\frac{\partial\left(\rho^{+} u_{k}^{+} u_{i}^{+}\right)}{\partial x_{k}^{+}}=-\frac{\partial P^{+}}{\partial x_{i}^{+}}+\frac{1}{R e} \frac{\partial\left(\tau_{k i}^{+}\right)}{\partial x_{k}^{+}}
\end{gathered}
$$




$$
\begin{aligned}
& \frac{\partial\left(\rho^{+} E^{+}\right)}{\partial t^{+}}+\frac{\partial\left(\rho^{+} u_{k}^{+} E^{+}\right)}{\partial x_{k}^{+}}=-(\gamma-1) M a^{2} \frac{\partial\left(P^{+} u_{k}^{+}\right)}{\partial x_{k}^{+}}+\frac{1}{R e}(\gamma-1) M a^{2} \frac{\partial\left(\tau_{k i}^{+} u_{i}^{+}\right)}{\partial x_{k}^{+}} \\
& +\frac{\tau}{\operatorname{Re} \operatorname{Pr}} \frac{\partial}{\partial x_{k}^{+}}\left[\lambda \frac{\partial T^{+}}{\partial x_{k}^{+}}\right]-\frac{\tau}{\operatorname{Re} \operatorname{Pr}} \frac{\partial}{\partial x_{k}^{+}}\left[\rho D \frac{\partial c}{\partial x_{k}^{+}}\right] \\
& \frac{\partial\left(\rho^{+} c\right)}{\partial t^{+}}+\frac{\partial\left(\rho^{+} u_{k}^{+} c\right)}{\partial x_{k}^{+}}=\dot{w}^{+}+\frac{1}{\operatorname{ReSc}} \frac{\partial}{\partial x_{k}^{+}}\left[\rho D \frac{\partial c}{\partial x_{k}^{+}}\right]
\end{aligned}
$$

where all the non-dimensional quantities are shown with a + in the superscript. In Eqs. B1-B4, Re $=\rho_{r e f} u_{r e f} L_{r e f} / \mu_{r e f}$ is the nominal Reynolds number, $M a=$ $u_{r e f} / a_{r e f}$ is the Mach number, $\gamma=C_{p} / C_{v}$ is the ratio of specific heats, $\operatorname{Pr}$ is the Prandtl number and $S c=P r \cdot L e$ is the Schmidt number with $\rho_{r e f}, u_{r e f}, L_{r e f}, a_{r e f}$ and $\mu_{r e f}$ are the reference values of density, velocity scale, length scale, acoustic velocity and viscosity respectively. Here the density, viscosity and acoustic speed of the unburned gas are taken to be $\rho_{r e f}, \mu_{r e f}$ and $a_{r e f}$ respectively, and $S_{L}$ and $10 \delta_{t h}$ are considered to be $u_{r e f}$ and $L_{r e f}$ respectively. The internal energy $E=C_{V} T+u_{k} u_{k} / 2+H(1-c)$ (where $H$ the heat of reaction per unit mass of reactants consumed) is normalised by $C_{p} T_{0}$ in the following manner:

$$
E^{+}=\frac{1}{\gamma}\left(1+\tau T^{+}\right)+\frac{1}{2}(\gamma-1) M a^{2} u_{k}^{+} u_{k}^{+}+\tau(1-c)
$$

where $T^{+}=\left(T-T_{0}\right) /\left(T_{a d}-T_{0}\right)$ is the non-dimensional temperature and $\tau=\left(T_{a d}-\right.$ $\left.T_{0}\right) / T_{0}$ is the heat release parameter with $T_{0}$ and $T_{a d}$ being the unburned and adiabatic flame temperatures respectively. The gas is assumed to follow the ideal gas law $P=\rho R T$ which takes the following non-dimensional form:

$$
P^{+}=\frac{1}{\gamma M a^{2}} \rho^{+}\left(1+\tau T^{+}\right)
$$

The normalised reaction rate of the reaction progress variable $c$ for single step Arrhenius type chemistry takes the following form:

$$
\dot{w}^{+}=B^{*} \rho^{+}(1-c) \exp \left[-\frac{\beta\left(1-T^{+}\right)}{1-\alpha\left(1-T^{+}\right)}\right]
$$

where $B^{*}$ is the normalised pre-exponential factor and $\alpha=\tau /(1+\tau)$ is the heat release parameter.

\section{References}

1. Bilger, R.W.: Some aspects of scalar dissipation. Flow Turb. Combust. 72, 93-114 (2004)

2. Knudsen, E., Richardson, E.S., Doran, E.M., Pitsch, H., Chen, J.H.: Modeling scalar dissipation and scalar variance in large eddy simulation: Algebraic and transport equation closures. Phys. Fluids 24, 055103 (2012)

3. Xia, J., Luo, K.H.: Direct numerical simulation of inert droplet effects on scalar dissipation rate in turbulent reacting and non-reacting shear layers. Flow Turbulence Combust. 84(3), 397-422 (2010) 
4. Mouangue, R., Obounou, M., Gomet, L., Mura, A.: Lagrangian intermittent modelling of a turbulent lifted methane-air jet flame stabilized in a vitiated air coflow. Flow Turbul. Combust. 92(3), 731-765 (2014)

5. Bray, K.N.C.: Turbulent flows with premixed reactants. In: Libby, P.A., Williams, F.A. (eds.): Turbulent reacting flows, pp. 115-183. Springer Verlag, Berlin Heidelburg (1980)

6. Dunstan, T., Minamoto, Y., Chakraborty, N., Swaminathan, N.: Scalar dissipation rate modelling for Large Eddy Simulation of turbulent premixed flames. Proc. Comb. Inst. 34, 1193-1201 (2013)

7. Gao, Y., Chakraborty, N., Swaminathan, N.: Algebraic closure of scalar dissipation rate for Large Eddy Simulations of turbulent premixed combustion. Combust. Sci. Technol. 186, 1309-1337 (2014)

8. Ma, T., Gao, Y., Kempf, A.M., Chakraborty, N.: Validation and implementation of algebraic les modelling of scalar dissipation rate for reaction rate closure in turbulent premixed combustion combust. Flame 161, 3134-3153 (2014)

9. Chakraborty, N., Cant, R.S.: Effects of Lewis number on Flame Surface Density transport in turbulent premixed combustion. Combust. Flame 158, 1768-1787 (2011)

10. Chakraborty, N., Swaminathan, N.: Effects of lewis number on scalar variance transport in premixed flames. Flow Turb. Combust. 87, 261-292 (2011)

11. Borghi, R., Dutoya, D.: On the scales of the fluctuations in turbulent combustion. Proc. Combust. Inst. 17, 235-244 (1978)

12. Borghi, R.: Turbulent premixed combustion: Further discussions on scales of fluctuations. Combust. Flame 80, 304-312 (1990)

13. Mantel, T., Borghi, R.: New model of premixed wrinkled flame propagation based on a scalar dissipation equation. Combust. Flame 96, 443-457 (1994)

14. Mura, A., Borghi, R.: Towards an extended scalar dissipation equation for turbulent premixed combustion. Combust. Flame 133, 193-196 (2003)

15. Swaminathan, N., Bray, K.N.C.: Effect of dilatation on scalar dissipation in turbulent premixed flames. Combust. Flame 143, 549-565 (2005)

16. Chakraborty, N., Swaminathan, N.: Influence of the Damköhler number on turbulence-scalar interaction in premixed flames. I. Physical insight. Phys. Fluids 19, 045103 (2007)

17. Chakraborty, N., Swaminathan, N.: Influence of the Damköhler number on turbulence-scalar interaction in premixed flames. II. Model development. Phys. Fluids 19, 045104 (2007)

18. Chakraborty, N., Rogerson, J.W., Swaminathan, N.: A priori assessment of closures for scalar dissipation rate transport in turbulent premixed flames using direct numerical simulation. Phys. Fluids 20, 045106 (2008)

19. Mura, A., Tsuboi, K., Hasegawa, T.: Modelling of the correlation between velocity and reactive scalar gradients in turbulent premixed flames based on DNS data. Combust. Theory Model. 12(4), 671-698 (2008)

20. Mura, A., Robin, V., Champion, M., Hasegawa, T.: Small-scale features of velocity and scalar fields of turbulent premixed flames. Flow Turb. Combust. 82, 339-358 (2009)

21. Mura, A., Champion, M.: Relevance of the Bray number in the small-scale modelling of turbulent premixed flames. Combust. Flame 150, 729-733 (2009)

22. Kolla, H., Rogerson, J.W., Chakraborty, N., Swaminathan, N.: Prediction of turbulent flame speed using scalar dissipation rate. Combust. Sci. Technol. 181, 518-535 (2009)

23. Chakraborty, N., Rogerson, J., Swaminathan, N.: The scalar gradient alignment statistics of flame kernels and its modelling implications for turbulent premixed combustion. Flow Turb. Combust. 85(1), 25-55 (2010)

24. Chakraborty, N., Swaminathan, N.: Effects of Lewis number on scalar dissipation transport and its modelling implications for turbulent premixed combustion. Combust. Sci. Technol. 182, 1201-1240 (2010)

25. Chakraborty, N., Champion, M., Mura, A., Swaminathan, N.: Scalar dissipation rate approach to reaction rate closure. In: Swaminathan, N., Bray, K.N.C. (eds.) Turbulent Premixed Flame. 1st Edition, pp. 76102. Cambridge University Press, Cambridge (2011)

26. Chakraborty, N., Swaminathan, N.: Reynolds number effects on scalar dissipation rate transport and its modelling in turbulent premixed combustion. Combust. Sci. Technol. 185, 676-709 (2013)

27. Dong, H.Q., Robin, V., Mura, A., Champion, M.: Analysis of algebraic closures of the mean scalar dissipation rate of the progress variable applied to stagnating turbulent flames. Flow Turbul. Combust. 90, 301-323 (2013)

28. Girimaji, S.S., Zhou, Y.: Analysis and modeling of subgrid scalar mixing using numerical data. Phys. Fluids 8(5), 1224 (1996)

29. Charlette, F., Meneveau, C., Veynante, D.: A power-law flame wrinkling model for LES of premixed turbulent combustion. Part II: Dynamic formulation Combust. Flame 131, 181-197 (2002) 
30. Charlette, F., Meneveau, C., Veynante, D.: A power law wrinkling model for LES of premixed turbulent combustion, Part I: Non dynamic formulation and initial tests. Combust. Flame 131, 159-180 (2002)

31. Knikker, R., Veynante, D., Meneveau, C.: A dynamic flame surface density model for large eddy simulations of turbulent premixed combustion. Phys. Fluids 16, 91-94 (2004)

32. Chakraborty, N., Klein, M.: A priori direct numerical simulation assessment of algebraic flame surface density models for turbulent premixed flames in the context of large eddy simulation. Phys. Fluids 20, 085108 (2008)

33. Katragadda, M., Chakraborty, N., Cant, R.S.: Effects of turbulent reynolds number on the performance of algebraic flame surface density models for large eddy simulation in the thin reaction zones regime: a direct numerical simulation analysis. J. Combust., 353257 (2012)

34. Katragadda, M., Chakraborty, N., Cant, R.S.: A-priori DNS assessment of wrinkling factor based algebraic Flame Surface Density models in the context of Large Eddy Simulations for non-unity Lewis number flames in the thin reaction zones regime. J. Combust. 794671 (2012)

35. Roberts, W.L., Driscoll, J.F., Drake, M.C., Goss, L.P.: Images of the quenching of a flame by a vortexTo quantify regimes of turbulent combustion. Combust. Flame 94, 58-69 (1993)

36. Pope, S.B.: Turbulent Flows, Cambridge University Press, UK, $1^{\text {st }}$ Edition (2000)

37. Pitsch, H., Duchamp, L.G.: Large-eddy simulation of turbulent premixed combustion using level-set approach. Proc. Combust. Inst. 29, 2001-2004 (2002)

38. Gao, Y., Chakraborty, N., Swaminathan, N.: Scalar dissipation rate transport in the context of large eddy simulations for turbulent premixed flames with non-unity lewis number. Flow Turb. Combust. 93, 461486 (2014)

39. Peters, N.: Turbulent combustion, 1st edn. Cambridge University Press, U.K (2000)

40. Chakraborty, N., Klein, M., Swaminathan, N.: Effects of Lewis number on reactive scalar gradient alignment with local strain rate in turbulent premixed flames. Proc. Combust. Inst. 32, 1409-1417 (2009)

41. Langella, I., Chakraborty, N., Swaminathan, N.: Large Eddy Simulations of premixed combustion using SDR approach", $8^{\text {th }}$ Mediterranean Combustion Symposium, Izmir, Turkey, $8^{\text {th }}-13^{\text {th }}$ September $(2013)$

42. Chen, J.H., Choudhary, A., De Supinski, B., DeVries, M., Hawkes, E.R., Klasky, S., Liao, W.K., Ma, K.L., Mellor-Crummey, J., Podhorski, N., Sankaran, R., Shende, S., Yoo, C.S.: Terascale direct numerical simulations of turbulent combustion using S3D. Comp. Sci. Discov. 2, 015001 (2009)

43. Richardson, E.S., Sankaran, R., Grout, R.W., Chen, J.H.: Numerical analysis of reaction-diffusion effects on species mixing rates in turbulent premixed methane-air combustion. Combust. Flame 157, 506-515 (2010)

44. Jenkins, K.W., Cant, R.S.: Proceedings of the 2nd AFOSR Conference on DNS and LES. In: Knight, D., Sakell, L. (eds.): Rutgers University. 191-202, Dordrecht (1999)

45. Chakraborty, N., Cant, S.: Unsteady effects of strain rate and curvature on turbulent premixed flames in an inlet-outlet configuration, Combust. Flame 137, 129-147 (2004)

46. Chakraborty, N., Hartung, G., Katragadda, M., Kaminski, C.F.: Comparison of 2D and 3D densityweighted displacement speed statistics and implications for laser based measurements of flame displacement speed using direct numerical simulation data. Combust. Flame 158, 1372-1390 (2011)

47. Chakraborty, N., Cant, R.S.: Turbulent reynolds number dependence of flame surface density transport in the context of reynolds averaged navier stokes simulations. Proc. Combust. Inst. 34, 1347-1356 (2013)

48. Chakraborty, N., Lipatnikov, A.N.: Conditional velocity statistics for high and low Damköhler number turbulent premixed combustion in the context of Reynolds Averaged Navier Stokes simulations. Proc. Combust. Inst. 34, 1333-1345 (2013)

49. Jiménez, C., Cuenot, B., Poinsot, T., Haworth, D.: Numerical simulation and modeling for lean stratified propane-air flames. Combust. Flame 128, 1-21 (2002)

50. Grout, R.W.: An age-extended progress variable for conditioning reaction rates. Phys. Fluids 19, 105107 (2007)

51. Han, I., Huh, K.H.: Effects of the Karlovitz number on the evolution of the flame surface density in turbulent premixed flames. Proc. Combust. Inst. 32, 1419-1425 (2009)

52. Pera, C., Chevillard, S., Reveillon, J.: Effects of residual burnt gas heterogeneity on early flame propagation and on cyclic variability in spark-ignited engines. Combust. Flame 160, 1020-1032 (2013)

53. Trouvé, A., Poinsot, T.: The evolution equation for flame surface density in turbulent premixed combustion. J. Fluid Mech. 278, 1-31 (1994)

54. Han, I., Huh, K.H.: Roles of displacement speed on evolution of flame surface density for different turbulent intensities and Lewis numbers in turbulent premixed combustion. Combust. Flame. 152, 194 205 (2008)

55. Muppala, S.R., Aluri, N.K., Dinkelacker, F., Leipertz, A.: Development of an algebraic reaction rate approach for the numerical calculation of turbulent premixed methane, ethylene and propane/air flames at pressures up to 1.0 MPa. Combust. Flame 140, 257-266 (2005) 
56. Chakraborty, N., Cant, R.S.: Direct numerical simulation analysis of the flame surface density transport equation in the context of large eddy simulation. Proc. Combust. Inst. 32, 1445-1453 (2009)

57. Sreenivasan, K.R.: Possible effects of small-scale intermittency in turbulent reacting flows. Flow Turbulence Combust. 72, 115-131 (2004)

58. Sreenivasan, K.R., Ramshankar, R., Meneveau, C.: Mixing, entrainment and fractal dimensions of surfaces in turbulent flows. Proc. R.Soc. Lond. A 421, 79-108 (1989)

59. Sreenivasan, K.R.: Fractals and multifractals in fluid turbulence. Annu. Rev. Fluid Mech. 23, 539-600 (1991)

60. Prasad, R.R., Sreenivasan, K.R.: Quantitative three-dimensional imaging and the structure of passive scalar fields in fully turbulent flows. J. Fluid Mech. 216, 1-34 (1990)

61. Shivamoggi, B.K.: Multi-fractal aspects of the fine-scale structure of temperature fluctuations in isotropic turbulence. Phys. A 221, 460-477 (1995)

62. Bardina, J., Ferziger, J., Reynolds, W.C.: Improved sub-grid models for large eddy simulation. AIAA J. 80, 1357 (1980)

63. Zang, Y., Street, R.L., Koseff, J.R.: A dynamic mixed subgrid-scale model and its application to turbulent recirculating flows. Phys. Fluids 5, 3186-3196 (1993)

64. Vreman, B., Geurts, B., Kuerten, H.: Large-eddy simulation of the turbulent mixing layer. J. Fluid Mech. 339, 357-390 (1997) 